\title{
The NANOGrav 11-year Data Set: Pulse Profile Variability
}

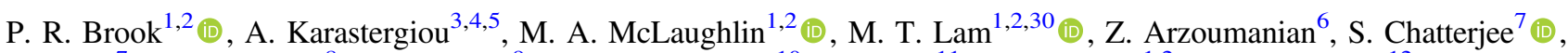

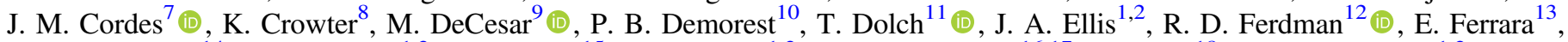

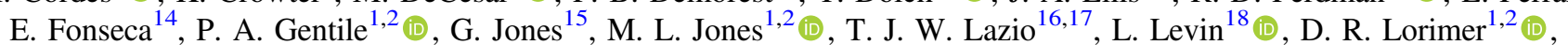

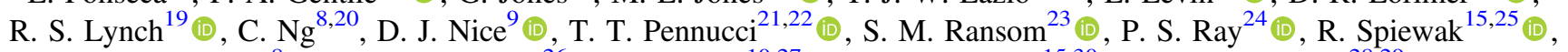

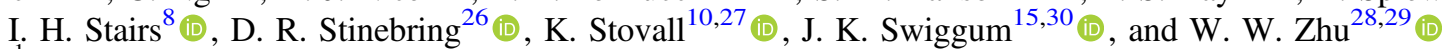

${ }^{1}$ Department of Physics and Astronomy, West Virginia University, Morgantown, WV 26506, USA; paul.brook@nanograv.org

${ }^{2}$ Center for Gravitational Waves and Cosmology, West Virginia University, Chestnut Ridge Research Building, Morgantown, WV 26505, USA

${ }^{3}$ Astrophysics, University of Oxford, Denys Wilkinson Building, Keble Road, Oxford OX1 3RH, UK ${ }_{4}^{4}$ Physics Department, University of the Western Cape, Cape Town 7535, South Africa

${ }^{5}$ Department of Physics and Electronics, Rhodes University, P.O. Box 94, Grahamstown 6140, South Africa

${ }^{6}$ X-Ray Astrophysics Laboratory, NASA Goddard Space Flight Center, Code 662, Greenbelt, MD 20771, USA

${ }_{8}^{7}$ Department of Astronomy and Cornell Center for Astrophysics and Planetary Science, Cornell University, Ithaca, NY 14853, USA

${ }^{8}$ Department of Physics and Astronomy, University of British Columbia, 6224 Agricultural Road, Vancouver, BC V6T 1Z1, Canada 9 Department of Physics, Lafayette College, Easton, PA 18042, USA

${ }_{11}^{10}$ National Radio Astronomy Observatory, 1003 Lopezville Road, Socorro, NM 87801, USA

${ }^{11}$ Department of Physics, Hillsdale College, 33 E. College Street, Hillsdale, MI 49242, USA

${ }^{12}$ Department of Physics, University of East Anglia, Norwich, NR4 7TJ, UK

${ }^{13}$ NASA Goddard Space Flight Center, Greenbelt, MD 20771, USA

${ }^{14}$ Department of Physics, Mcgill University, 3600 Rue University, Montreal, QC H3A 2T8, Canada

${ }^{15}$ Center for Gravitation, Cosmology and Astrophysics, Department of Physics, University of Wisconsin-Milwaukee, P.O. Box 413, Milwaukee, WI 53201, USA

${ }^{16}$ Jet Propulsion Laboratory, California Institute of Technology, 4800 Oak Grove Drive, Pasadena, CA 91109, USA

${ }^{17}$ Theoretical AstroPhysics Including Relativity (TAPIR), MC 350-17, California Institute of Technology, Pasadena, CA 91125, USA

18 Jodrell Bank Centre for Astrophysics, Alan Turing Building, School of Physics and Astronomy,

The University of Manchester, Oxford Road, Manchester, M13 9PL, UK

${ }^{19}$ Green Bank Observatory, P.O. Box 2, Green Bank, WV 24944, USA

${ }^{20}$ Dunlap Institute for Astronomy \& Astrophysics, University of Toronto, 50 St. George Street, Toronto, ON M5S 3H4, Canada

${ }^{21}$ Eötvös Loránd University, Budapest, Hungary

${ }^{22}$ Hungarian Academy of Sciences MTA-ELTE Extragalactic Astrophysics Research Group, 1117 Budapest, Hungary

${ }^{23}$ National Radio Astronomy Observatory, 520 Edgemont Road, Charlottesville, VA 22903, USA

${ }^{24}$ Naval Research Laboratory, Washington DC 20375, USA

${ }^{25}$ Centre for Astrophysics and Supercomputing, Swinburne University of Technology, P.O. Box 218, Hawthorn VIC 3122, Australia

${ }^{26}$ Department of Physics \& Astronomy, Oberlin College, Oberlin, OH 44074, USA

${ }^{27}$ Department of Physics and Astronomy, University of New Mexico, Albuquerque, NM 87131, USA

${ }^{28}$ National Astronomical Observatories, Chinese Academy of Science, 20A Datun Road, Chaoyang District, Beijing 100012, People's Republic of China

${ }^{29}$ Max-Planck-Institut für Radioastronomie, Auf dem Hügel 69, D-53121, Bonn, Germany

Received 2018 April 21; revised 2018 September 17; accepted 2018 October 17; published 2018 November 30

\begin{abstract}
Access to 50 years of data has led to the discovery of pulsar emission and rotation variability on timescales of months and years. Most of this long-term variability has been seen in long-period pulsars, with relatively little focus on recycled millisecond pulsars. We have analyzed a 38-pulsar subset of the 45 millisecond pulsars in the NANOGrav 11-year data set, in order to review their pulse profile stability. The most variability, on any timescale, is seen in PSRs J1713+0747, B1937+21, and J2145-0750. The strongest evidence for long-timescale pulse profile changes is seen in PSRs B1937+21 and J1643-1224. We have focused our analyses on these four pulsars in an attempt to elucidate the causes of their profile variability. Effects of scintillation seem to be responsible for the profile modifications of PSR J2145-0750. We see evidence that imperfect polarization calibration contributes to the profile variability of PSRs J1713+0747 and B1937+21, along with radio frequency interference around $2 \mathrm{GHz}$, but find that propagation effects also have an influence. The changes seen in PSR J1643-1224 have been reported previously, yet elude explanation beyond their astrophysical nature. Regardless of cause, unmodeled pulse profile changes are detrimental to the accuracy of pulsar timing and must be incorporated into the timing models where possible.
\end{abstract}

Key words: ISM: general - pulsars: general - pulsars: individual (J1643-1224, J1713+0747, B1937+21, J2145 -0750) - stars: neutron

\section{Introduction}

The radio emission from a pulsar can vary over a wide range of timescales. In practically all pulsars, the rotational phase, shape, and amplitude of individual radio pulses are known to vary considerably from one to the next with each rotation (e.g.,

\footnotetext{
${ }^{30}$ NANOGrav Physics Frontiers Center Postdoctoral Fellow.
}

Lyne et al. 1971; Taylor et al. 1975). The average shape of a few thousand pulses, however, is typically very stable and known as the pulse profile (e.g., Helfand et al. 1975; Rathnasree \& Rankin 1995). Soon after pulsars were discovered, however, changes in some pulse profiles were seen on short timescales in the form of mode-changing and nulling (Backer 1970a, 1970b). Mode-changing is a phenomenon in which pulsars switch between two or more quasi-stable 
emission states on timescales ranging from a few pulse periods to hours and days. In a nulling pulsar, one of these states shows little or no emission.

Pulsar data have now been collected for more than 50 years. This allows us to also identify longer-term pulse profile variability. In 2006, the first known intermittent pulsar was identified by Kramer et al. (2006). Intermittent pulsars go through a quasi-periodic cycle between phases in which radio emission is, and is not, detected. The timescale of this behavior ranges from months to years (Camilo et al. 2012; Lorimer et al. 2012; Lyne et al. 2017). In intermittent pulsars, each state is associated with a different rate of rotational velocity loss, known as the spindown rate $\dot{\nu}$. Kramer et al. attribute $\dot{\nu}$ variations to global changes in magnetospheric particle currents; changing numbers of charged particles at the polar cap would simultaneously affect the pulsar's radio emission. More links between pulse profile and rotation were provided in Lyne et al. (2010), an analysis that showed six pulsars for which $\dot{\nu}$ is correlated with changes in pulse shape over months and years. Further notable examples of long-term variability, including pulse profile and spindown correlation, continue to be found (e.g., Brook et al. 2014, 2016). All of the examples of long-term pulse profile variability provided are found in longperiod pulsars (typically defined as those with spin periods above around $30 \mathrm{~ms}$ and those that have not been spun up or recycled through the accretion of matter from a companion star); relatively little work has been done regarding the longterm pulse profile variability of millisecond pulsars (MSPs). The issue of stability for MSPs is particularly important, however, as they are employed as high-precision timing tools that can facilitate fundamental studies of physics. For example, MSPs are used in pulsar timing arrays in an attempt to detect gravitational waves at nanohertz frequencies (Hobbs 2013; Kramer \& Champion 2013; McLaughlin 2013). MSPs are suitable for this role, as they are known to be more rotationally stable than long-period pulsars due to their high angular momentum. Additionally, the time of arrival (TOA) of a pulse from an MSP can be measured with more precision than that of a long-period pulsar, as the uncertainty is proportional to the temporal width of a pulsar's pulse profile.

A pulse TOA is measured by a process of template matching (Taylor 1992; van Straten 2006). The stability of a pulse profile at a given frequency permits the cross-correlation of an observed profile with a high signal-to-noise ratio $(\mathrm{S} / \mathrm{N})$ template, to provide a TOA of the former. The template is either the average of many previous observations, or a noisefree model of this average. Therefore, any unmodeled pulse profile changes will result in inaccurate pulse TOAs, which are detrimental to an MSP's utility as a timing tool.

Pulse profile variability can be caused by any of the following: intrinsic changes in the pulsar and/or its magnetosphere, geodetic precession (Kramer 1998; Hotan et al. 2005), torque-free precession (Stairs et al. 2000), propagation through the ionized interstellar medium (IISM), instrumental effects, and radio frequency interference (RFI). In addition to the potential benefits for pulsar timing, understanding the causes of pulse profile variability and the sometimes correlated changes in rotational behavior may elucidate physical processes intrinsic to pulsars and their magnetosphere and also constrain the effects of pulse propagation.

As the long-term pulse profile variability of MSPs has not been well studied, it has only previously been reported in the
MSP J1643-1224; Shannon et al. (2016) describe a sudden and permanent broadband pulse profile modification, accompanied by changes in timing.

In Brook et al. (2016), new techniques were used to identify pulse profile variability in long-period pulsar data collected by the Parkes Telescope. In this work we apply similar techniques to a large sample of MSPs using data recorded by the NANOGrav collaboration, with the aim of uncovering and quantifying MSP pulse profile variability. The NANOGrav collaboration produces TOAs by template matching the pulse profile in each frequency channel (typically between 5 and 64 over the observing band; Arzoumanian et al. 2015), thereby producing multiple TOAs for each observation. The analysis done here, however, looks for changes in pulse profiles that have been frequency-integrated over the observing band. This is done to maximize the $\mathrm{S} / \mathrm{N}$ to facilitate the principal aim of characterizing the long-term profile behavior in the pulsar. However, when integrating a pulsar signal over a wide observing band, pulse profiles are more susceptible to variations induced by propagation effects (e.g., Pennucci et al. 2014).

In Section 2 we describe the NANOGrav data used for the variability analysis outlined in Section 3 . The results of the analysis are presented in Section 4, followed by a discussion in Section 5. Conclusions are drawn in Section 6.

\section{Data}

The data analyzed in this paper are a subset of the NANOGrav 11-year data set (Arzoumanian et al. 2018), collected by the Green Bank Telescope (GBT) and Arecibo Observatory (AO). Since 2010, data collected by the GBT have been recorded by the Green Bank Ultimate Pulsar Processing Instrument (GUPPI; DuPlain et al. 2008; Ford et al. 2010). The observations are carried out at center frequencies around 820 and $1500 \mathrm{MHz}$. Since 2012, data collected at AO have been recorded by the Puerto Rican Ultimate Pulsar Processing Instrument (PUPPI). The observations are carried out at center frequencies around $327 \mathrm{MHz}$ (PSR J2317+1439 only), and 430, 1400, and $2030 \mathrm{MHz}$. This GUPPI/PUPPI subset was used, as the instruments process a bandwidth of up to $800 \mathrm{MHz}$ (divided into $1.5625 \mathrm{MHz}$ frequency channels), depending on the mode of operation. Details of frequency coverage are given in Table 1 of Arzoumanian et al. (2015). Earlier narrowbandwidth data in the NANOGrav data set were excluded from this analysis due to relatively low $\mathrm{S} / \mathrm{N}$.

GUPPI and PUPPI performed coherent dedispersion and folding in real-time. The data were folded at the dynamically calculated pulsar period using a pre-computed ephemeris to produce the pulse profile, consisting of 2048 phase bins. The pulsar signals were flux and polarization calibrated, and narrow-band RFI was removed in the manner of Arzoumanian et al. (2018).

The polarization calibration was done via an injected calibration signal that is generated by a local noise diode at $25 \mathrm{~Hz}$. Preceding each pulsar observation, the noise diode signal is split, coupled into the two polarization paths, and measured with the pulsar backends. This permits calibration of the differential gain and phase between the two hands of polarization. For a complete description of the instrumental response to a polarized signal, one must compute the Mueller matrix: a frequency-dependent linear transformation from the intrinsic to observed Stokes parameters (Heiles et al. 2001; 
van Straten 2004). The Mueller matrix is determined by tracking a polarized source over a wide range of parallactic angles and fitting the resulting variation of the observed Stokes parameters as the feed rotates with respect to the sky. This allows the determination of effects such as the magnitude and phase of the cross coupling of the receiver arms.

While all the data sets have undergone noise diode calibration as described, full Mueller matrix calibration has also been performed on the $1500 \mathrm{MHz}$ GUPPI data only. As this method provides more accurate pulse profile information, the GUPPI $1500 \mathrm{MHz}$ profiles analyzed in this work have had full Mueller matrix calibration applied, unless stated otherwise. Mueller matrix calibration has also recently been applied to PUPPI data by Gentile et al. (2018), but their results have not been included in this analysis. The pulsed noise signals themselves were calibrated in on- and off-source observations of unpolarized continuum radio sources on a monthly basis.

Each final pulse profile analyzed here is the integration of typically 20-30 minutes of observation across the entire frequency band. Pulsars at declinations between $0^{\circ}$ and $+39^{\circ}$ were observed at $\mathrm{AO}$, while all others were observed with the GBT. PSRs J1713+0747 and B1937+21 were observed with both telescopes.

The dispersion measure (DM) is fit to the data at almost every observing epoch and applies for a window of up to 14 days, though typically much shorter. In NANOGrav timing analysis, an additional timing delay $\Delta t_{\mathrm{FD}}$ is added to all timing models to compensate for TOA perturbations induced by the frequency-dependence of pulse profile shapes. DM and $\Delta t_{\mathrm{FD}}$ are covariant when finding the best-fit timing model parameters for a pulsar, and so the best-fit DM value is highly dependent on $\Delta t_{\mathrm{FD}}$. For the purposes of creating the frequency-integrated pulse profiles employed in this variability analysis, we have calculated the best-fit DM parameters without the inclusion of $\Delta t_{\mathrm{FD}}$. This is discussed further in Section 5.4.

Further details of the observations, data reduction, and timing models can be found in Arzoumanian et al. (2018) and references therein.

\section{Analysis}

The most effective metric for quantifying pulse profile variability is dependent on the timescales involved. Pulsar observations can often be widely and irregularly spaced; smooth trends that occur on timescales much longer than the time between these observations may not be obvious when analyzing individual pulse profiles. Such trends can instead be revealed when the variability is modeled and interpolated across many epochs of observation. If the pulse profile variations take place on timescales comparable to or shorter than the span between observations, then any variability may appear stochastic and a smooth trend (if one is present) may not be easily detected. The analysis techniques used to uncover and quantify both of these systematic and noisy types of pulse profile variability are described in the following discussion.

To quantify the amount of pulse profile variability in an individual observation, we calculate the differences between the observed pulse profile and a constant model; these differences are termed the profile residuals (Brook et al. 2016). The model for a particular pulsar and observing frequency is a median profile; a median value is calculated in each individual phase bin using all observations in the pulsar data set. The median was used so that the model would be minimally affected by any outlying pulse profile shapes. The technique used to align the profiles before constructing the median model is simple cross-correlation. We note that the shape of the model is not crucial, as we are interested in how the observations change with time. The model merely defines the zero-point for the profile residuals.

Before the profile residuals can be calculated, the observations are processed to ensure that the off-pulse baseline is centered on zero. Any individual observations with highly irregular pulse profiles are treated as the result of RFI or instrumental issues and removed from further analysis. Additionally, the noisiest observations in a data set are considered unreliable and also excluded; an observation is removed if the standard deviation of the off-pulse region is more than a factor of two larger than the median value taken from the off-pulse regions across all epochs.

Pulse profile changes can manifest as a modulation of shape or as a change in flux density across the profile as a whole. Large flux density variations are observed in most of the pulsar data analyzed in this work, and are thought to be attributable to refractive and diffractive interstellar scintillation (RISS and DISS, respectively; e.g., Rickett 1990). To disentangle the less common pulse profile shape changes, we must normalize the flux density of all observations. Alignment of the profiles with the constant model is also essential for the analysis that follows, as the timeseries in each pulse phase bin are modeled independently. This alignment is non-trivial; when profile deviations occur (either intrinsically or due to effects of propagation, instrumentation or RFI), it is possible that the alignment may be slightly biased in that direction when simple cross-correlation is employed. In this analysis, the flux density normalization and the phase alignment are carried out simultaneously in the following way.

\subsection{Flux Density Normalization and Phase Alignment}

In order to compare pulse profile shapes, we need to align them in phase and normalize them in flux density as effectively as possible. In many cases, the TOAs deviate enough from the pulsar timing model to disqualify their use in the alignment of the observations. Traditional profile alignment and normalization algorithms use $\chi^{2}$ minimization techniques and operate on all profile bins. These algorithms are susceptible to biases in cases when the two profiles differ in shape over some range of pulse phase. For this reason, we employed the following robust fitting algorithm, which is less susceptible to such biases. We characterize two pulse profiles as being correctly normalized and aligned by maximizing the number of phase bins that are in agreement; this is defined more formally as follows. Each observation, in turn, is normalized and aligned relative to the constant model. The observed profile is shifted in phase over the model. For each of the 2048 phase bin alignments, the scaling factor of the observation is varied over a range defined such that the observation's profile peak is within $10 \%$ of the peak of the constant model. This range is sampled uniformly in 100 steps. The $10 \%$ restriction will reduce computation time while safely accommodating all realistic scaling trials. For each combination of phase shift and scaling factor, the absolute difference between model and observation is calculated in each phase bin along with the mean,

$$
\delta=\frac{1}{n} \sum_{i}^{n}\left|d_{i}-m_{i}\right|,
$$




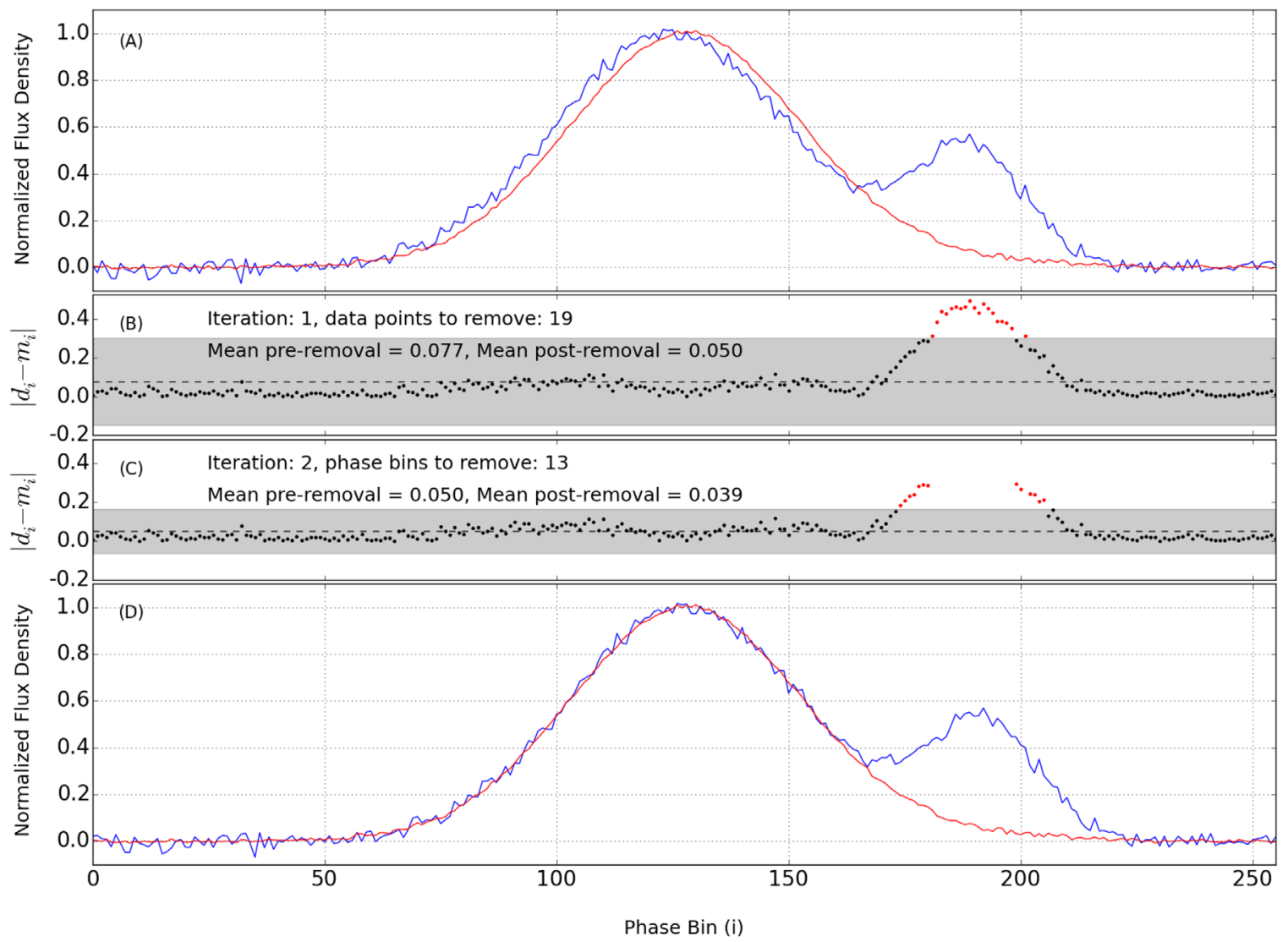

Figure 1. Method used to normalize and align pulse profiles. Panel (A) shows a simulated comparison of a model profile (red) and an observed profile (blue) with a trailing edge deviation. The alignment shown as an example is the result of simple cross-correlation. For every alignment, a series of iterations, such as those illustrated in Panels (B) and (C), are executed until a value for $\delta / n_{\mathrm{f}}$ is eventually determined (see text for details). In Panel (B), the black dots show the absolute difference between the observed and model profiles in each phase bin $\left(\left|d_{i}-m_{i}\right|\right)$. The dashed line indicates the mean and the gray band shows two standard deviations of the data; any points outside this band are removed before the standard deviation is recalculated and the process is repeated, as seen in Panel (C). For each iteration, the mean of the data is calculated before and after the data removal. A change by less than $0.1 \%$ signifies the end of the process (14 iterations were required for this outlier removal in these simulated data shown in Panel (A)). The last data to be removed are restored and the final mean $\delta$ of $\left|d_{i}-m_{i}\right|$ is calculated. This value, divided by the number of contributing phase bins $\delta / n_{\mathrm{f}}$, is minimized while adjusting the phase and normalized flux density of the observation (relative to the model). Panel (D) is the relative alignment with the lowest value of $\delta / n_{\mathrm{f}}$.

where $d_{i}$ and $m_{i}$ are the values of the observational data and the model (respectively) in phase bin $i$, and $n$ is the number of phase bins in the calculation. For identical profile shapes, for example, $\delta$ will be zero as the two profiles overlay exactly. We next exclude any phase bins in which $\left|d_{i}-m_{i}\right|$ is more than two standard deviations $(2 \sigma)$ away from $\delta$. After these outliers are removed, $\delta$ is then recalculated. This step is repeated until the recalculated mean $\delta$ changes by less than $0.1 \%$ of its previous value, at which stage the phase bin exclusion process is considered complete. The final number of phase bins that have not been excluded is $n_{\mathrm{f}}$. These steps are illustrated in Figure 1. All remaining bins now have relatively comparable values of $\left|d_{i}-m_{i}\right|$. This process is performed so that only the stable parts of the profile are used to align and scale - that is, localized profile deviations that appear in observations are not required to match the constant model. To align and scale the profiles, we want to minimize the differences between the non-excluded phase bins, but also want to penalize fits in which only a small number of phase bins remain after the exclusion process. In order to find the optimal fit, we minimize $\delta / n_{\mathrm{f}}$.

In this analysis we calculate the variability of both normalized and non-normalized pulse profiles. The latter are also aligned using the technique provided, but their flux density levels are restored at the end of the process.
Precision timing of pulsars demands that observations are aligned to fractions of a phase bin (under the assumption that the pulse profile is unchanging). However, aligning in single bin increments (with 2048 bin resolution) is simple and sufficient in this profile profile variability analysis; any profile residuals produced by fractional phase bin misalignment would typically be insignificant when compared to the amount of noise in individual bins. If required, higher precision alignment could be implemented.

Once the pulse profiles are correctly aligned and normalized, we can proceed to calculate and analyze the profile residuals.

\subsection{Visualizing Variability}

In Brook et al. (2016), a technique was developed that models pulse profiles as a function of time, allowing interpolation between the epochs of observation. For each of the pulse profile phase bins, we computed a Gaussian process (GP) regression model that best describes the profile residuals (Rasmussen 2006; Roberts et al. 2012). The lengthscale hyperparameter for the GP regression models was constrained to between 30 and 300 days for every data set analyzed; we find that this requirement results in the data being well represented by the models. Full details of the GP regression analysis can be found in Brook et al. (2016). Examples of this 

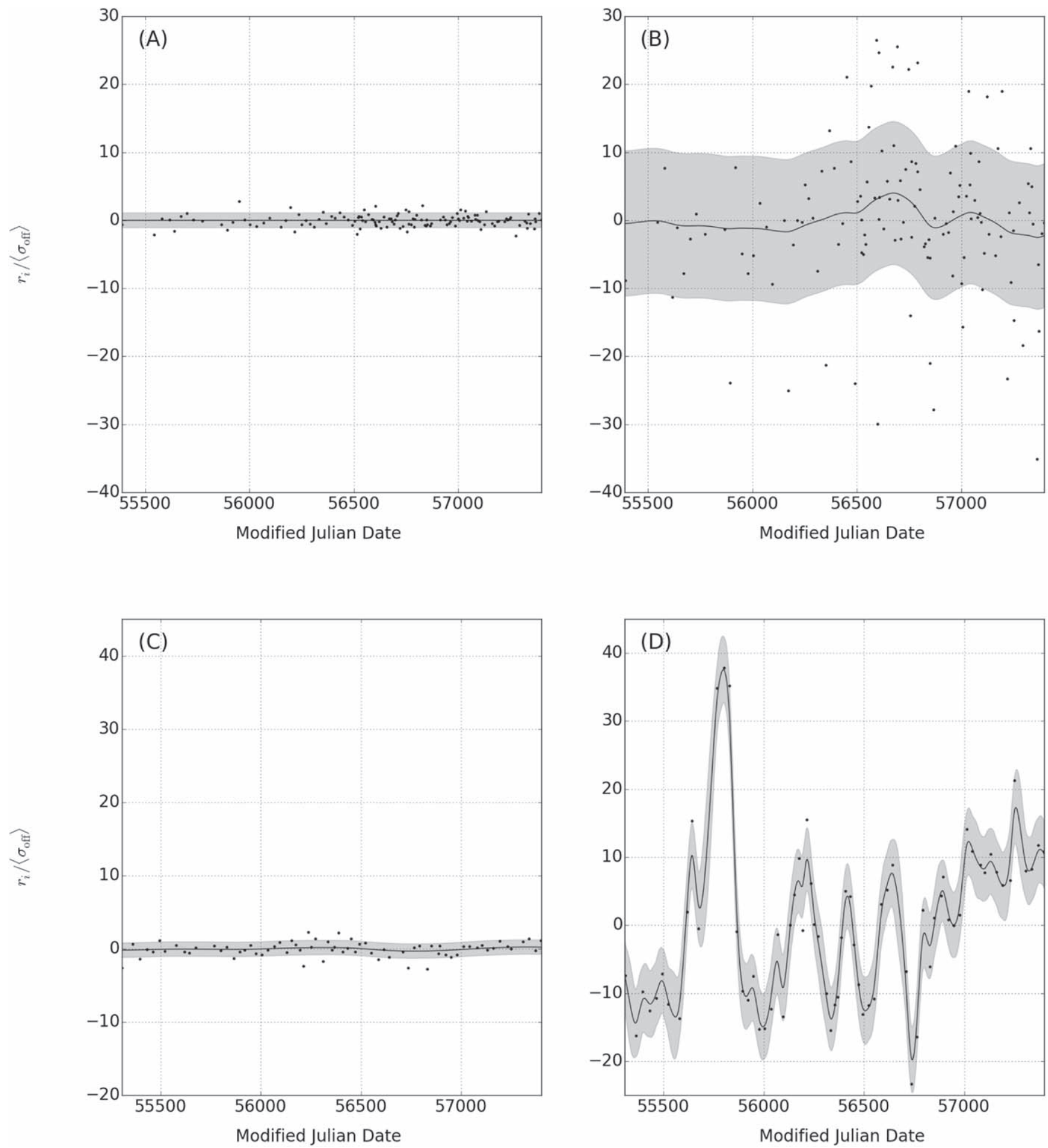

Figure 2. Profile residuals and their GP models. Panel (A) and Panel (B) show data from example off- and on-pulse phase bins, respectively, for PSR J1713+0747 at an observation frequency of $1500 \mathrm{MHz}$. Panels (C) and (D) show data from example off- and on-pulse bins, respectively, for PSR B1937+21 at an observational frequency of $820 \mathrm{MHz}$. In each panel, the data points are the profile residuals for a single phase bin, the solid black line shows the GP mean, and the gray area shows the GP standard deviation across the phase bin data set. Panel (B) is an example of a phase bin containing predominantly noisy data (with some systematic behavior also embedded within), whereas the data in Panel (D) show a very clearly systematic trend, with relatively little noise. $r_{i}$ is the profile residual of bin $i$; $\left\langle\sigma_{\text {off }}\right\rangle$ is the mean of the off-pulse profile residual standard deviation.

inference technique are shown in Figure 2. The individual phase bin models can be combined to produce a variability map for each pulsar. This is an interpolated plot that smoothly maps the evolution of a pulsar's profile residuals with time. The GP regression technique can be used to identify subtle long-term trends that are not visible by eye, as demonstrated in Brook et al. (2016). For each pulsar discussed in Section 4, a variability map was produced for both pre- and post-normalization pulse profiles, so that the flux density of the observations can be compared with any profile shape changes seen. 
The two pulsars in Figure 2 illustrate two different types of pulse profile variability (as mentioned at the beginning of Section 3). The systematic nature of the profile residuals in Panel (D) is well modeled by GP regression; the extent and nature of the profile variability is, therefore, easily captured by a variability map. In contrast, Panel (B) shows that the profile residuals in the $\mathrm{J} 1713+0747$ on-pulse phase bin are highly variable over time, but primarily in a noisy rather than systematic way. As a consequence, the GP model may infer little or no systematic variability and simply lie around the mean of the data points that inform it. The gray band in each Figure 2 panel shows the standard deviation of the model, however, and so provides an alternative measure of pulse profile variability. In order to show the amount of noisy variability for a pulsar data set, we also generate a color map showing the standard deviation of the GP model as a function of pulse phase and time. For instructional purposes, the color maps for a stable pulsar data set are shown in the Appendix (Figure 25).

\subsection{Quantifying Variability}

For each pulsar data set analyzed, we computed six metrics to fully describe the nature of the variability observed in the normalized pulse profiles. The metrics are defined as follows:

(A) Ratio of (i) mean standard deviation of on-pulse phase bins to (ii) mean standard deviation of off-pulse phase bins: $\left\langle\sigma_{\text {on }}\right\rangle /\left\langle\sigma_{\text {off }}\right\rangle$.

To calculate $\left\langle\sigma_{\mathrm{on}}\right\rangle$, we found the standard deviation of the profile residuals in each on-pulse phase bin and then calculated the mean across all epochs. The equivalent calculation was done for $\left\langle\sigma_{\text {off }}\right\rangle$.

(B) Ratio of (i) maximum standard deviation of on-pulse phase bins to (ii) mean standard deviation of off-pulse phase bins: $\sigma_{\text {on, max }} /\left\langle\sigma_{\text {off }}\right\rangle$.

The maximum standard deviation of the profile residuals in individual on-pulse phase bins provides information regarding any variability that may be concentrated over a small section of the pulse profile.

(C) Ratio of (i) peak systematic variability to (ii) mean standard deviation of off-pulse phase bins: $M_{\max } /\left\langle\sigma_{\text {off }}\right\rangle$.

$M_{\max }$ is the peak value of the GP model over all onpulse phase bins.

(D) Ratio of (i) average systematic variability to (ii) mean standard deviation of off-pulse phase bins: $\langle|M|\rangle /\left\langle\sigma_{\text {off }}\right\rangle$.

$\langle|M|\rangle$ is the mean of the absolute value of the GP model for on-pulse phase bins.

(E) Ratio of (i) noisy variability to (ii) mean standard deviation of off-pulse phase bins: $\left\langle\sigma_{\mathrm{M}}\right\rangle /\left\langle\sigma_{\text {off }}\right\rangle$.

$\left\langle\sigma_{\mathrm{M}}\right\rangle$ is the mean of the standard deviation of the GP model (i.e., the gray shaded regions in Figure 2) across all on-pulse phase bins. In pulsars with systematic variability (e.g., Panel (D) of Figure 2), the standard deviation about the GP model mean will be less than the standard deviation of the data themselves.

(F) Ratio of (i) average systematic variability to (ii) noisy variability: $\langle|M|\rangle /\left\langle\sigma_{\mathrm{M}}\right\rangle$.

This metric is indicative of the amount of long-term, systematic variability in a data set.

An on-pulse phase bin is defined as one in which the flux density of the median profile for the data set is more than $3 \%$ of the peak. An off-pulse phase bin is defined as one in which the flux density of the median profile for the data set is less than
$0.1 \%$ of the peak. The $3 \%$ and $0.1 \%$ values were chosen empirically to reliably select only on- and off-pulse phase bins, respectively. The gap between the thresholds exists in order to avoid contamination between the two. If on- and off-pulse variability is comparable, metrics $A$ and $E$ will have a value around unity.

\section{Results}

The results of the pulse profile variability analysis are presented in Table 1, which is ordered by pulsar right ascension and then by observing frequency. Only the NANOGrav data sets that consist of 20 or more observations (after noisy and unreliable profiles are removed) are featured in the table. This is done to ensure that the GP regression has sufficient data points to infer an accurate model; 78 data sets (from a 38-pulsar subset of the 45 pulsars observed in the NANOGrav 11-year data set) remain after this requirement. The vast majority of pulsars show relatively little variability, with the mean standard deviation of their on-pulse phase bins being less than a factor of two greater than that of their off-pulse bins (Metric A of Table 1). The three pulsars for which this factor is greatest (denoted by an asterisk in Table 1) are PSRs J1713+0747, B1937+21, and J2145-0750. We have selected these pulsars for further analysis, and later we discuss the nature and possible causes of the profile variability for each of them. In addition, we also focus on PSR J1643-1224; after PSR B1937+21, the $820 \mathrm{MHz}$ data set for this pulsar (denoted by a double asterisk in Table 1) has the largest average systematic to noisy variability ratio (Metric $F$ of Table 1), which indicates the presence of long-term variability. PSR J1643-1224 has also previously demonstrated unusual chromatic timing behavior and long-term pulse profile shape changes (Shannon et al. 2016).

\subsection{PSR $J 1713+0747$}

Due in part to the high $\mathrm{S} / \mathrm{N}$ of its pulse profile, PSR J1713 +0747 is one of the most precisely timed pulsars. Arzoumanian et al. (2018) list the standard deviation of the epoch-averaged timing residuals (the differences between observed TOAs and a timing model) for this pulsar as $116 \mathrm{~ns}$ over 11 years of NANOGrav observations. The high $\mathrm{S} / \mathrm{N}$ also allows any pulse profile variations to be seen clearly.

The $1400 \mathrm{MHz}$ AO observations of PSR J1713+0747 display the most profile variability of all data sets analyzed in this work; Table 1 shows that this is mostly noisy in nature (a relatively large value for variability metric $\mathrm{E}$ and a relatively small value for variability metric F). Despite the average systematic variability of the data set being low with respect to the noisy variability, the peak of the systematic variability is high; the GP model is being strongly affected over short time periods by three observations with anomalous profile shapes (MJDs 56360, 56598, and 57239). This can be seen in the variability map in Panel (B3) of Figure 3. These three profiles are compared to those typical for the data set in Figure 4. For the observation made on MJD 56360, it is known that during the flux density calibration procedure, an incorrect pulsed calibration signal was injected at the epoch of observation. It is not clear whether the pulse profile shape was affected by this. However, no such calibration issues exist for the observations made on MJDs 56598 or 57239 . In the "Discussion" section, we compare these three profiles to those expected to be 
Table 1

The Variability Calculated in 78 NANOGrav MSP Data Sets

\begin{tabular}{|c|c|c|c|c|c|c|c|}
\hline $\begin{array}{l}\text { Pulsar } \\
\text { Name }\end{array}$ & $\begin{array}{c}\text { Observing } \\
\text { Frequency } \\
(\mathrm{MHz})\end{array}$ & $\left\langle\sigma_{\mathrm{on}}\right\rangle /\left\langle\sigma_{\mathrm{off}}\right\rangle$ & $\sigma_{\text {on,max }} /\left\langle\sigma_{\text {off }}\right\rangle$ & $\begin{array}{c}M_{\max } /\left\langle\sigma_{\mathrm{off}}\right\rangle \\
\text { (C) }\end{array}$ & $\langle|M|\rangle /\left\langle\sigma_{\text {off }}\right\rangle$ & $\begin{array}{c}\left\langle\sigma_{\mathrm{M}}\right\rangle /\left\langle\sigma_{\text {off }}\right\rangle \\
(\mathrm{E})\end{array}$ & $\begin{array}{c}\langle|M|\rangle /\left\langle\sigma_{\mathrm{M}}\right\rangle \\
(\mathrm{F})\end{array}$ \\
\hline $\mathrm{J} 0023+0923$ & 430 & 1.13 & 1.97 & 3.58 & 0.12 & 1.10 & 0.11 \\
\hline $\mathrm{J} 0023+0923$ & 1400 & 1.17 & 2.74 & 3.69 & 0.08 & 1.17 & 0.07 \\
\hline $\mathrm{J} 0030+0451$ & 430 & 1.02 & 1.76 & 2.81 & 0.06 & 1.02 & 0.06 \\
\hline $\mathrm{J} 0030+0451$ & 1400 & 1.03 & 1.72 & 1.97 & 0.05 & 1.03 & 0.05 \\
\hline $\mathrm{J} 0340+4130$ & 820 & 1.03 & 1.70 & 1.95 & 0.08 & 1.02 & 0.08 \\
\hline $\mathrm{J} 0340+4130$ & 1500 & 1.001 .00 & 1.421 .38 & 3.073 .44 & $\begin{array}{lll}0.09 & 0.09\end{array}$ & $\begin{array}{lll}0.98 & 0.99\end{array}$ & $\begin{array}{lll}0.09 & 0.09\end{array}$ \\
\hline J0613-0200 & 820 & 1.08 & 3.01 & 3.56 & 0.11 & 1.06 & 0.10 \\
\hline J0613-0200 & 1500 & 1.041 .04 & $1.47 \quad 1.70$ & 3.923 .16 & $\begin{array}{ll}0.08 & 0.08\end{array}$ & $1.03 \quad 1.03$ & $\begin{array}{lll}0.08 & 0.08\end{array}$ \\
\hline $\mathrm{J} 0636+5128$ & 820 & 1.10 & 1.634 .16 & 0.14 & 1.07 & 0.13 & \\
\hline $\mathrm{J} 0636+5128$ & 1500 & $1.08 \quad 1.07$ & 1.741 .64 & 4.343 .54 & 0.100 .13 & 1.061 .02 & $\begin{array}{lll}0.10 & 0.13\end{array}$ \\
\hline $\mathrm{J} 0645+5158$ & 820 & 1.06 & 2.30 & 3.47 & 0.09 & 1.04 & 0.09 \\
\hline $\mathrm{J} 0645+5158$ & 1500 & 1.061 .05 & 2.092 .12 & 5.965 .72 & $\begin{array}{lll}0.10 & 0.12\end{array}$ & 1.031 .02 & $0.10 \quad 0.12$ \\
\hline J0931 - 1902 & 820 & 1.03 & 1.71 & 4.14 & 0.11 & 1.01 & 0.11 \\
\hline J0931 - 1902 & 1500 & 1.091 .05 & $2.08 \quad 1.87$ & 3.733 .79 & $\begin{array}{ll}0.11 & 0.12\end{array}$ & $1.08 \quad 1.02$ & $\begin{array}{ll}0.10 & 0.12\end{array}$ \\
\hline $\mathrm{J} 1012+5307$ & 820 & 1.09 & 1.77 & 3.57 & 0.12 & 1.07 & 0.11 \\
\hline $\mathrm{J} 1012+5307$ & 1500 & $1.13 \quad 1.12$ & 2.922 .64 & 3.392 .56 & 0.060 .09 & 1.121 .11 & 0.050 .08 \\
\hline $\mathrm{J} 1024-0719$ & 820 & 1.06 & 1.72 & 3.59 & 0.09 & 1.04 & 0.09 \\
\hline $\mathrm{J} 1024-0719$ & 1500 & 1.051 .06 & $1.71 \quad 1.80$ & 3.563 .31 & $\begin{array}{ll}0.10 & 0.08\end{array}$ & $1.03 \quad 1.05$ & $\begin{array}{ll}0.10 & 0.08\end{array}$ \\
\hline $\mathrm{J} 1125+7819$ & 820 & 1.04 & 1.71 & 4.21 & 0.15 & 0.99 & 0.15 \\
\hline $\mathrm{J} 1455-3330$ & 820 & 1.10 & 2.04 & 3.44 & 0.11 & 1.09 & 0.10 \\
\hline $\mathrm{J} 1455-3330$ & 1500 & 1.091 .07 & 3.002 .71 & 5.756 .23 & 0.160 .15 & 1.041 .02 & 0.150 .15 \\
\hline $\mathrm{J} 1600-3053$ & 820 & 1.05 & 1.40 & 2.36 & 0.09 & 1.04 & 0.09 \\
\hline $\mathrm{J} 1600-3053$ & 1500 & 1.151 .24 & 2.102 .96 & 1.996 .31 & $0.08 \quad 0.10$ & 1.141 .23 & $\begin{array}{lll}0.07 & 0.08\end{array}$ \\
\hline $\mathrm{J} 1614-2230$ & 820 & 1.02 & 1.39 & 2.99 & 0.09 & 1.00 & 0.09 \\
\hline $\mathrm{J} 1614-2230$ & 1500 & $1.01 \quad 1.00$ & 1.291 .33 & 2.591 .91 & 0.050 .06 & 1.001 .00 & 0.050 .06 \\
\hline $\mathrm{J} 1640+2224$ & 430 & 1.07 & 1.63 & 2.57 & 0.04 & 1.07 & 0.04 \\
\hline $\mathrm{J} 1640+2224$ & 1400 & 1.32 & 2.85 & 2.75 & 0.08 & 1.32 & 0.06 \\
\hline${ }^{* *} \mathrm{~J} 1643-1224$ & 820 & 1.14 & 1.75 & 3.40 & 0.29 & 1.05 & 0.28 \\
\hline $\mathrm{J} 1643-1224$ & 1500 & $1.01 \quad 1.00$ & 1.421 .32 & 2.332 .26 & $\begin{array}{lll}0.09 & 0.09\end{array}$ & 1.000 .99 & $\begin{array}{lll}0.09 & 0.09\end{array}$ \\
\hline $\mathrm{J} 1713+0747$ & 820 & 1.36 & 5.11 & 3.33 & 0.12 & 1.35 & 0.09 \\
\hline${ }^{*} \mathrm{~J} 1713+0747$ & 1400 & 8.78 & 52.19 & 269.88 & 0.89 & 8.65 & 0.10 \\
\hline $\mathrm{J} 1713+0747$ & 1500 & $2.38 \quad 2.21$ & $12.47 \quad 11.54$ & 6.856 .23 & $\begin{array}{lll}0.12 & 0.27\end{array}$ & 2.362 .18 & $\begin{array}{ll}0.05 & 0.12\end{array}$ \\
\hline $\mathrm{J} 1713+0747$ & 2030 & 3.36 & 11.58 & 4.08 & 0.10 & 3.39 & 0.03 \\
\hline $\mathrm{J} 1738+0333$ & 1400 & 1.34 & 6.00 & 5.88 & 0.14 & 1.29 & 0.11 \\
\hline $\mathrm{J} 1741+1351$ & 430 & 1.22 & 3.09 & 3.70 & 0.16 & 1.17 & 0.14 \\
\hline $\mathrm{J} 1741+1351$ & 1400 & 1.11 & 2.37 & 3.52 & 0.10 & 1.10 & 0.09 \\
\hline J1744-1134 & 820 & 1.12 & 1.56 & 3.00 & 0.11 & 1.10 & 0.10 \\
\hline $\mathrm{J} 1744-1134$ & 1500 & $1.38 \quad 1.35$ & 2.262 .45 & 2.413 .71 & $\begin{array}{lll}0.11 & 0.11\end{array}$ & $1.37 \quad 1.34$ & $\begin{array}{lll}0.08 & 0.08\end{array}$ \\
\hline $\mathrm{J} 1747-4036$ & 820 & 1.03 & 1.68 & 2.44 & 0.12 & 1.01 & 0.12 \\
\hline $\mathrm{J} 1747-4036$ & 1500 & 1.031 .03 & 1.521 .62 & 3.052 .97 & 0.120 .11 & $1.01 \quad 1.01$ & 0.120 .11 \\
\hline $\mathrm{J} 1832-0836$ & 820 & 1.02 & 1.55 & 2.69 & 0.09 & 1.01 & 0.09 \\
\hline $\mathrm{J} 1832-0836$ & 1500 & 1.031 .03 & 1.491 .66 & 3.044 .91 & 0.090 .10 & $1.01 \quad 1.00$ & $\begin{array}{lll}0.09 & 0.10\end{array}$ \\
\hline $\mathrm{J} 1853+1303$ & 430 & 1.06 & 1.82 & 2.85 & 0.08 & 1.06 & 0.08 \\
\hline $\mathrm{J} 1853+1303$ & 1400 & 1.07 & 1.66 & 4.46 & 0.10 & 1.05 & 0.10 \\
\hline B $1855+09$ & 430 & 1.03 & 1.46 & 3.61 & 0.08 & 1.02 & 0.08 \\
\hline B1855+09 & 1400 & 1.25 & 3.77 & 3.98 & 0.11 & 1.24 & 0.09 \\
\hline $\mathrm{J} 1903+0327$ & 1400 & 1.10 & 1.75 & 3.19 & 0.21 & 1.05 & 0.20 \\
\hline $\mathrm{J} 1903+0327$ & 2030 & 1.10 & 2.00 & 4.90 & 0.13 & 1.08 & 0.12 \\
\hline J1909-3744 & 820 & 1.59 & 2.95 & 3.65 & 0.24 & 1.54 & 0.16 \\
\hline J1909-3744 & 1500 & 1.651 .58 & 3.122 .81 & $1.51 \quad 1.74$ & $\begin{array}{lll}0.04 & 0.07\end{array}$ & 1.651 .58 & $\begin{array}{ll}0.02 & 0.04\end{array}$ \\
\hline $\mathrm{J} 1910+1256$ & 1400 & 1.03 & 1.59 & 2.89 & 0.08 & 1.02 & 0.08 \\
\hline $\mathrm{J} 1910+1256$ & 2030 & 1.04 & 1.58 & 4.76 & 0.17 & 0.99 & 0.17 \\
\hline J1918-0642 & 820 & 1.03 & 1.42 & 1.96 & 0.08 & 1.02 & 0.08 \\
\hline J1918-0642 & 1500 & 1.051 .05 & $1.58 \quad 1.54$ & 1.923 .04 & 0.050 .06 & 1.051 .04 & 0.050 .06 \\
\hline $\mathrm{J} 1923+2515$ & 430 & 1.06 & 2.00 & 2.93 & 0.09 & 1.05 & 0.09 \\
\hline $\mathrm{J} 1923+2515$ & 1400 & 1.09 & 1.95 & 5.11 & 0.10 & 1.07 & 0.09 \\
\hline${ }^{*} \mathrm{~B} 1937+21$ & 820 & 5.35 & 12.20 & 37.01 & 2.69 & 3.60 & 0.75 \\
\hline B1937+21 & 1400 & 5.09 & 12.86 & 23.42 & 0.91 & 4.82 & 0.19 \\
\hline B $1937+21$ & 1500 & 5.073 .36 & 10.857 .76 & 3.823 .24 & 0.420 .39 & 5.053 .33 & $\begin{array}{lll}0.08 & 0.12\end{array}$ \\
\hline B1937+21 & 2030 & 2.93 & 8.30 & 14.32 & 1.31 & 2.36 & 0.56 \\
\hline J1944+0907 & 430 & 1.09 & 1.96 & 3.38 & 0.10 & 1.08 & 0.09 \\
\hline $\mathrm{J} 1944+0907$ & 1400 & 1.12 & 2.06 & 3.91 & 0.13 & 1.09 & 0.12 \\
\hline
\end{tabular}


Table 1

(Continued)

\begin{tabular}{|c|c|c|c|c|c|c|c|}
\hline $\begin{array}{l}\text { Pulsar } \\
\text { Name }\end{array}$ & $\begin{array}{c}\text { Observing } \\
\text { Frequency } \\
(\mathrm{MHz})\end{array}$ & $\begin{array}{c}\left\langle\sigma_{\text {on }}\right\rangle /\left\langle\sigma_{\text {off }}\right\rangle \\
\text { (A) }\end{array}$ & $\begin{array}{c}\sigma_{\text {on,max }} /\left\langle\sigma_{\text {off }}\right\rangle \\
\text { (B) }\end{array}$ & $\begin{array}{c}M_{\max } /\left\langle\sigma_{\text {off }}\right\rangle \\
\text { (C) }\end{array}$ & $\begin{array}{c}\langle|M|\rangle /\left\langle\sigma_{\text {off }}\right\rangle \\
\text { (D) }\end{array}$ & $\begin{array}{c}\left\langle\sigma_{\mathrm{M}}\right\rangle /\left\langle\sigma_{\text {off }}\right\rangle \\
\text { (E) }\end{array}$ & $\begin{array}{c}\langle|M|\rangle /\left\langle\sigma_{\mathrm{M}}\right\rangle \\
(\mathrm{F})\end{array}$ \\
\hline B1953+29 & 430 & 1.10 & 1.91 & 4.22 & 0.22 & 1.03 & 0.21 \\
\hline $\mathrm{J} 2010-1323$ & 820 & 1.14 & 2.76 & 3.59 & 0.09 & 1.12 & 0.08 \\
\hline $\mathrm{J} 2010-1323$ & 1500 & 1.101 .10 & $2.33 \quad 2.42$ & $2.80 \quad 2.37$ & $0.06 \quad 0.07$ & 1.091 .10 & $0.06 \quad 0.06$ \\
\hline $\mathrm{J} 2017+0603$ & 1400 & 1.11 & 5.55 & 5.47 & 0.15 & 1.08 & 0.14 \\
\hline $\mathrm{J} 2043+1711$ & 1400 & 1.04 & 1.74 & 5.11 & 0.10 & 1.02 & 0.10 \\
\hline${ }^{*} \mathrm{~J} 2145-0750$ & 820 & 1.76 & 8.62 & 3.79 & 0.31 & 1.72 & 0.18 \\
\hline $\mathrm{J} 2145-0750$ & 1500 & $1.37 \quad 1.31$ & 3.023 .51 & 4.693 .82 & 0.220 .15 & $1.32 \quad 1.28$ & $\begin{array}{ll}0.17 & 0.12\end{array}$ \\
\hline $\mathrm{J} 2214+3000$ & 1400 & 1.00 & 1.73 & 5.37 & 0.12 & 0.97 & 0.12 \\
\hline $\mathrm{J} 2302+4442$ & 820 & 1.02 & 1.57 & 2.34 & 0.07 & 1.01 & 0.07 \\
\hline $\mathrm{J} 2302+4442$ & 1500 & 1.041 .00 & 1.541 .56 & 4.732 .66 & $\begin{array}{lll}0.10 & 0.09\end{array}$ & $\begin{array}{ll}1.01 & 0.99\end{array}$ & $\begin{array}{ll}0.10 & 0.09\end{array}$ \\
\hline
\end{tabular}

Note. An asterisk denotes each of the three data sets with the highest values for the ratio of the mean standard deviation of on- to off-pulse phase bins (Metric A; a measurement of the level of profile variability of any kind). These data are from the PSRs J1713+0747, B1937+21, and J2145-0750. Highlighted with a double asterisk is the 820 MHz data set for PSR J1643-1224, which has the highest ratio of average systematic to noisy variability (Metric F; a measurement of the significance of systematic variability) after PSR B1937+21. Each of the six variability metrics is described in Section 3.3. The data sets observed with the GBT at $1500 \mathrm{MHz}$ have two values for each variability metric. The left of the pair relates to profiles calibrated by the noise diode, and the right to profiles that additionally have full Mueller matrix calibration applied (see Section 2).
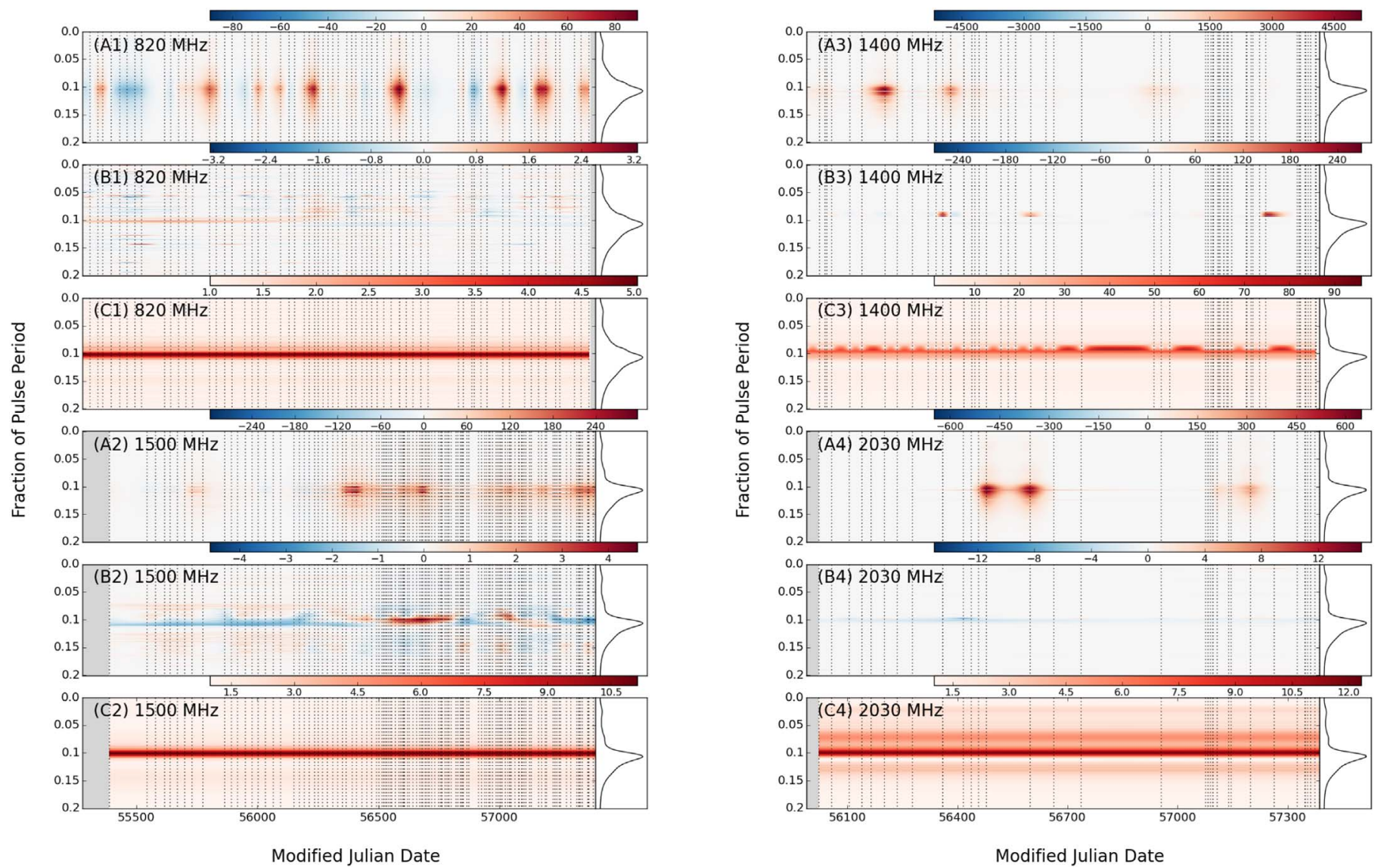

Figure 3. Variability maps for PSR J1713+0747, for central observing frequencies of $820 \mathrm{MHz}$ (GBT), $1500 \mathrm{MHz}$ (GBT), $1400 \mathrm{MHz}$ (AO), and 2030 MHz (AO). Panel labels prefixed with an (A) are variability maps showing the flux density variations in the flux-calibrated, pre-normalized observations. Those prefixed with a (B) show pulse profile shape changes after the observations have been normalized. In all of these variability maps, red regions indicate where the inferred pulse profile has an excess of flux density (normalized or otherwise) compared to the average for the data set. Blue indicates where it has a deficit. Panel labels prefixed with a (C) map the standard deviation of the GP model as a function of pulse phase and time. The vertical dotted lines indicate the epochs of observation informing the GP models. The unit for all panels is the mean of the standard deviation of the off-pulse phase bins for the relevant data set. Panel sections for which there is no data are gray. To the right of each panel, the average pulse profile for the data set is shown. 


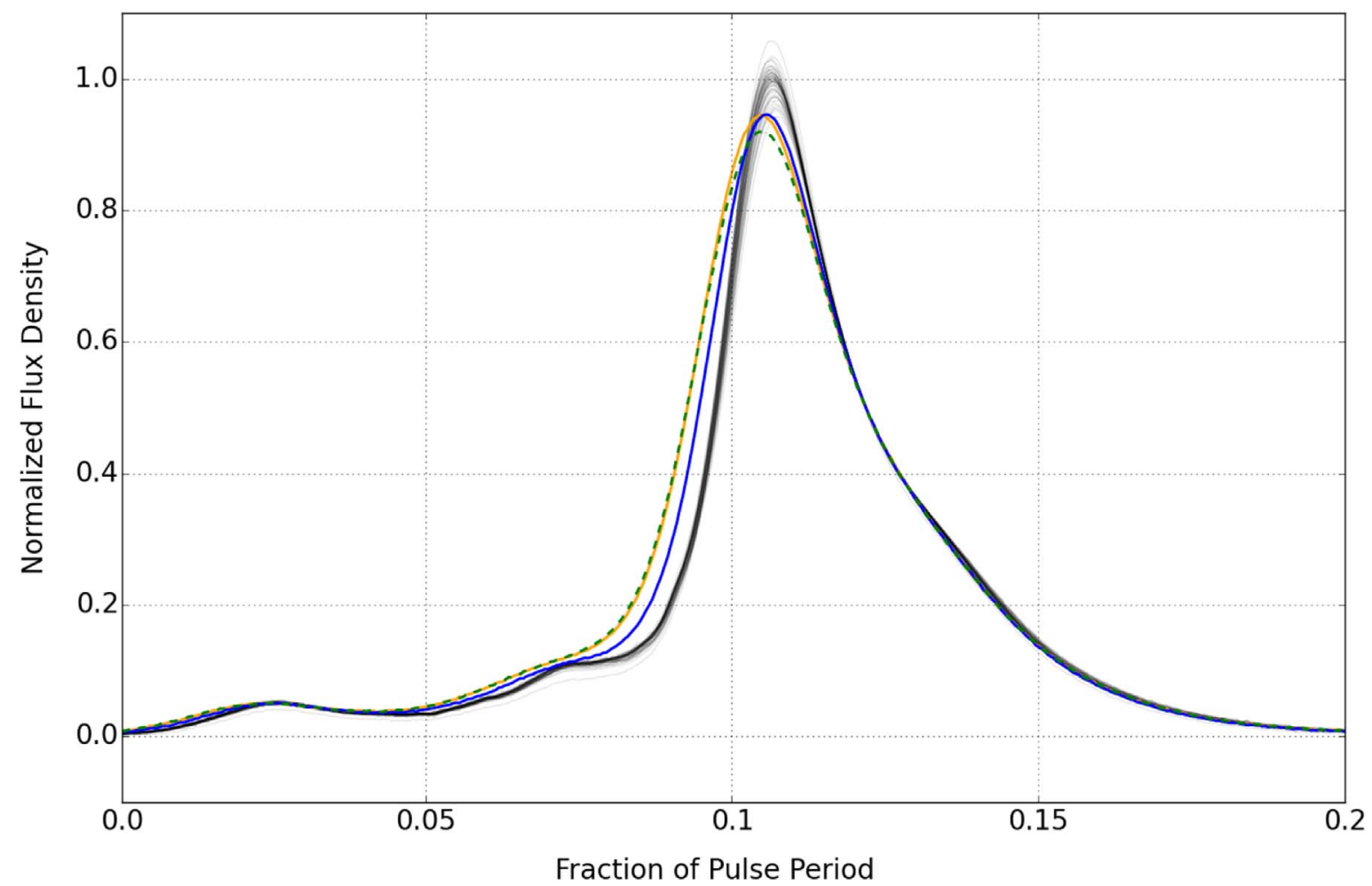

Figure 4. Pulse profile variability seen in PSR J1713+0747 at $1400 \mathrm{MHz}$. The orange, blue, and green (dashed) profiles were observed on MJDs 56360 , 56598 , and 57239 , respectively. The gray profiles show the other 58 pulse profiles in the data set. See also Figure 18 and Section 5.4 for a comparison of these three noteworthy observations to profile changes caused by inaccurate DMs in this data set.

produced by inaccurate DM measurements. This is shown in Figure 4 and described in Section 5.4.

The observations made at $2030 \mathrm{MHz}$ show a high degree of noisy variability, particularly in the latter half of the data set; the ratio of average systematic to noisy variability $\langle|M|\rangle /\left\langle\sigma_{\mathrm{M}}\right\rangle$ is the smallest of all data sets. The ratio of the mean standard deviation of the on- to off-pulse phase bins $\left\langle\sigma_{\text {on }}\right\rangle /\left\langle\sigma_{\text {off }}\right\rangle$ is not as large as that of the $1400 \mathrm{MHz}$ data set, however, because at $2030 \mathrm{MHz},\left\langle\sigma_{\text {off }}\right\rangle$ is much larger.

All PSR J1713+0747 data sets are dominated by noisy variability (i.e., profile shape changes largely occur on timescales shorter than the time between observations), and so the noisy variability far exceeds any systematic variability modeled by the GP. Some systematic variability is present, however; the PSR J1713+0747 data set with the highest ratio of average systematic to noisy variability (Metric $\mathrm{F}$ of Table 1) is recorded at $1500 \mathrm{MHz}$. Panel (B) of Figure 2 depicts the systematic behavior of the GP model (embedded in the primarily noisy variability) in an individual phase bin for the data set. The bin is associated with a pulse period fraction of $\sim 0.1$ in Panel (B2) of Figure 3.

As the variability in the PSR J1713+0747 data sets is predominantly short-term in nature, it is difficult to compare even the pulse profiles that were observed at similar frequencies, unless they are also observed at the same time. The $1400 \mathrm{MHz}$ AO and $1500 \mathrm{MHz}$ GBT observations are often made just days apart, but only simultaneous observations could permit us to observe identical pulse profile shapes and allow us to confirm the nature of any profile variability seen, as astrophysical.

\subsection{PSR $B 1937+21$}

PSR B1937+21 was the first MSP discovered (Backer et al. 1982) and, with a rotational frequency of $642 \mathrm{~Hz}$, remained the most rapidly spinning pulsar known for 24 years after it was found. This bright, isolated MSP is one of the most precisely timed pulsars, with the root mean square (rms) value of the white noise component for the 11-year data set residuals being $109 \mathrm{~ns}$ (Arzoumanian et al. 2018). It is also, however, one of the few MSPs that displays measurable timing noise (Shannon \& Cordes 2010). Including both the red and white noise components of the timing residuals, the rms calculated by Arzoumanian et al. jumps up to $1.5 \mu \mathrm{s}$. Suggested interpretations of the red noise include intrinsic changes in the spindown rate of the pulsar (Kaspi et al. 1994), interstellar propagation effects (Armstrong 1984; Rickett 1990; Kaspi et al. 1994; Cognard et al. 1995), and the presence of a circumpulsar asteroid belt (Shannon et al. 2013). PSR B1937+21 has also been seen to exhibit giant pulses; around one in every 10,000 individual pulses has more than 20 times the mean on-pulse flux density, and some pulses have around 300 times this average (Cognard et al. 1996). This behavior is seen in both the main and interpulse components, which are separated by approximately half a pulse period. In our pulse profile analysis, PSR B1937+21 shows the most systematic variability, present primarily at 820 and $2030 \mathrm{MHz}$ and in both the main and interpulse components. This can be seen in Metric F of Table 1, where the ratios of systematic to noisy variability for PSR B1937+21 are much higher than those for PSR J1713 +0747 . Panels (B1) and (B4) of Figures 5 and 6 also clearly highlight the systematic evolution of the pulse profile shape 

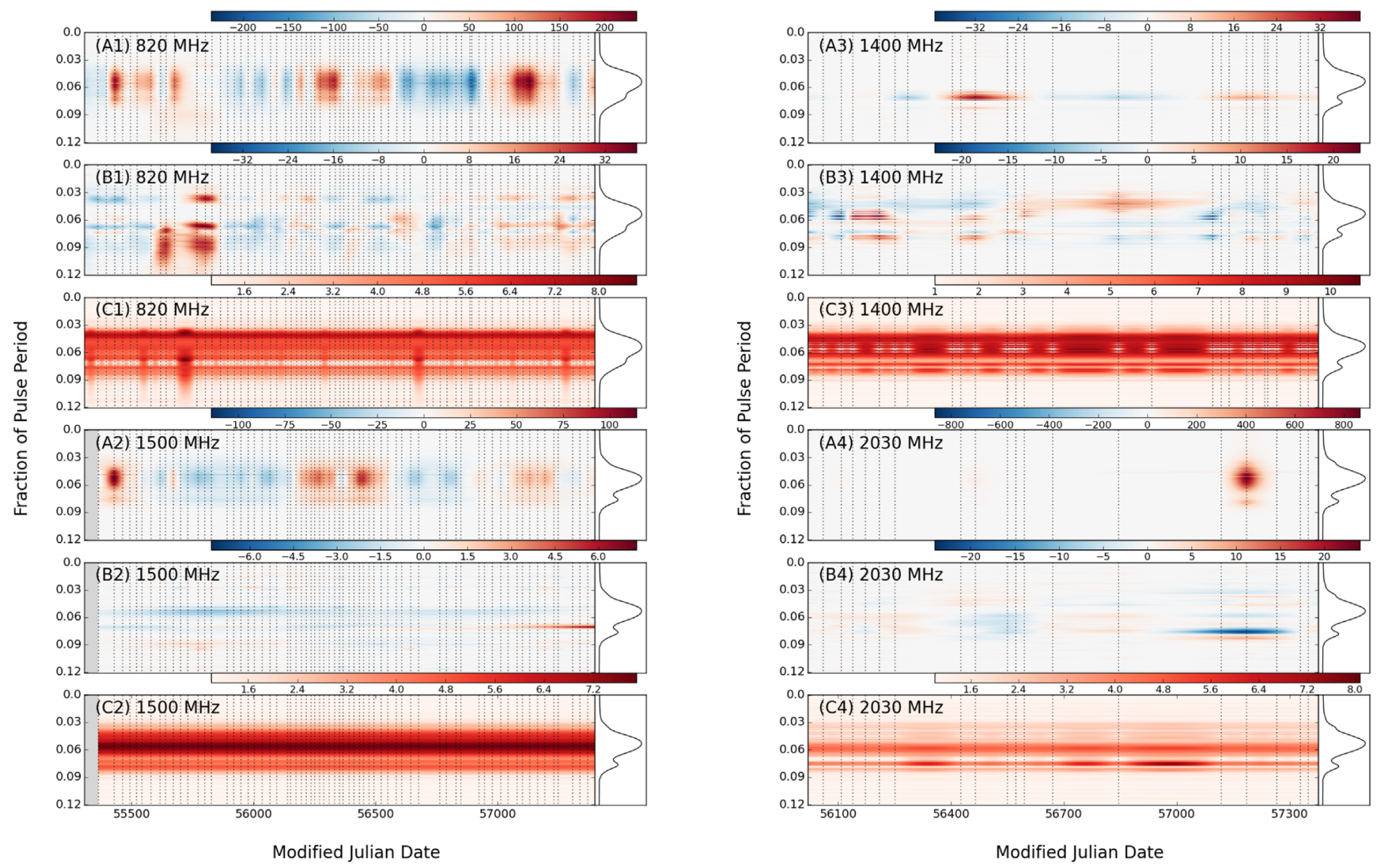

Figure 5. Variability maps for the main pulse of PSR B1937+21. Otherwise, this figure is the same as Figure 3.

over time. The variability inferred by the GP model at $2030 \mathrm{MHz}$ between $\sim \mathrm{MJDs} 57000$ and 57300 (see Panel (B4) of Figures 5 and 6) is induced by three consecutive pulse profiles. They are compared with the rest of the profiles in the data set in Figure 7. See also the discussion around polarization calibration in Section 5.6 and Figure 23.

A direct comparison between the profile variability seen by $\mathrm{AO}$ at $1400 \mathrm{MHz}$ and by GBT at $1500 \mathrm{MHz}$ is made difficult primarily because the observations at this frequency have the smallest ratio of systematic to noisy variability of the PSR B1937+21 data sets (Metric F of Table 1). Therefore, much of the variability is noisy, but only longer timescale systematic trends can be directly compared, as the observations are generally not made on the same days. Some systematic structure appears in the $1500 \mathrm{MHz}$ GBT observation (Panel (B2) of Figures 5 and 6), but the units of these panels show that the systematic variability is weak, with the GP model reaching levels only a few times higher than the levels of off-pulse noise. At such levels, the GP model can be substantially influenced by the behavior of even one or two pulse profiles. Additionally, the GBT and AO data sets analyzed here span different dates; much of the systematic variability in the $1500 \mathrm{MHz}$ GBT observations occurs around MJD 56000, which is before the $1400 \mathrm{MHz}$ AO data were recorded by PUPPI. Furthermore, the relatively sparse sampling of the $\mathrm{AO}$ observations also inhibits direct comparison with the GBT GP variability models.

\subsection{PSR J2145-0750}

PSR J2145-0750 has the third highest variability levels (by the metric in Metric A of Table 1) of the pulsars in our analysis. It is found to have a mean standard deviation of on-pulse phase bins that is a factor of 1.76 larger than that of the off-pulse phase bins (at $820 \mathrm{MHz}$ ). As with PSR J1713+0747, most of the variability is noisy; Panel (B1) of Figure 8 shows a long-timescale change in the pulse profile shape, but the magnitude of this change is small compared to the standard deviation of the data (Panel (C1)).

\subsection{PSR J1643-1224}

PSR J1643-1224 has been observed since 2003 as part of the Parkes Pulsar Timing Array project (Manchester et al. 2013) at 700, 1400, and $3100 \mathrm{MHz}$. Shannon et al. (2016) noticed TOA perturbations, which they attributed to unmodeled changes in pulse shape, observed to occur around MJD 57074 (2015 February 21). These timing perturbations are most significant at $3100 \mathrm{MHz}$, and Shannon et al. only show profile changes at that frequency. Figure 9 provides a comparison of the pulse profile shapes of PSR J1643-1224 before and after 2015 February 21, as observed by the GBT. The upper panels show a significant difference at $820 \mathrm{MHz}$, but little change at $1500 \mathrm{MHz}$. Shannon et al. (2016) compare the shape variations of PSR J1643-1224 to those observed in PSR J0738-4042 (Karastergiou et al. 2011) and also point out that the new components are unpolarized in both pulsars. A new component is seen to appear in PSR J0738-4042 after a drifting feature is observed to move centrally over a span of $\sim 100$ days (Brook et al. 2014). The variability map in Panel (B1) of Figure 10 shows that changes in the profile shape appear to be occurring across the data set, and not just abruptly after MJD 57074. In particular, red colored drifting features can be seen at the beginning and end of the data set. The drifting at the end of the 

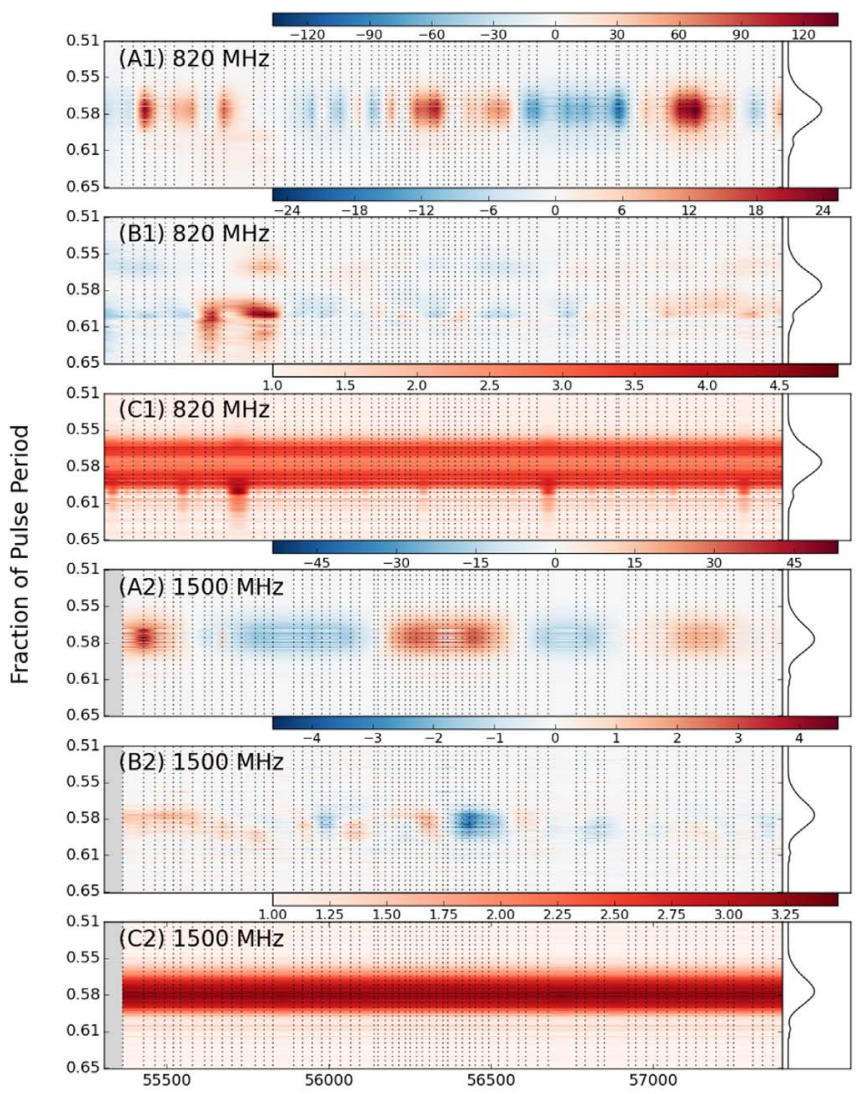

Modified Julian Date

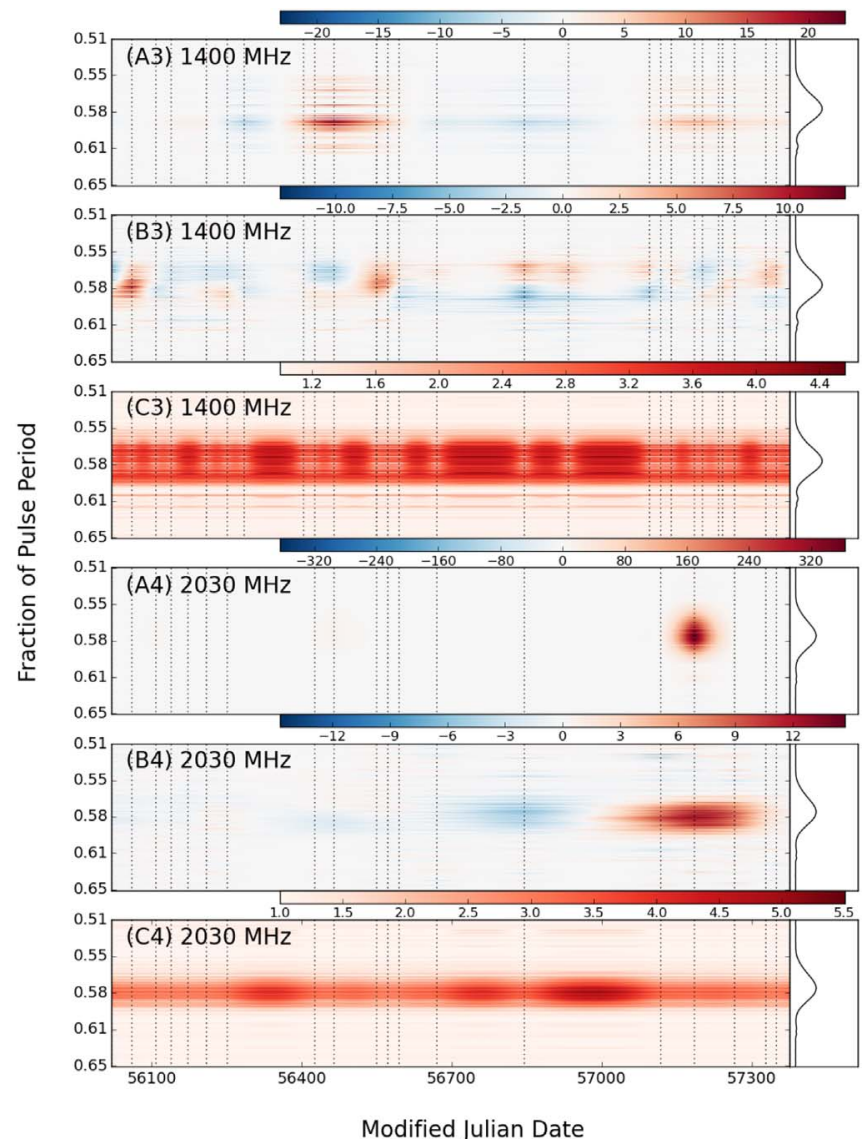

Figure 6. Variability maps for the interpulse of PSR B1937+21. Otherwise, this figure is the same as Figure 3.

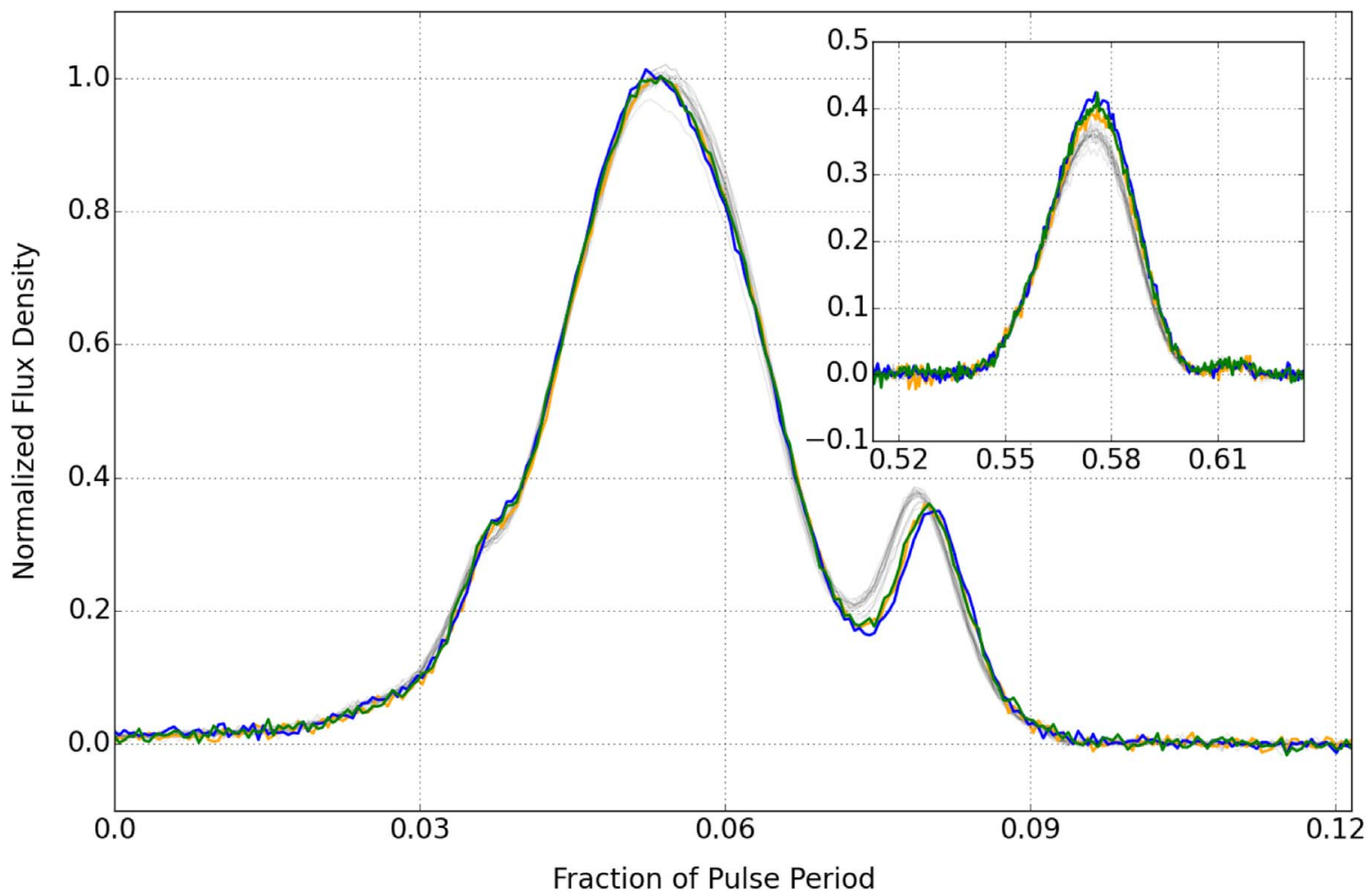

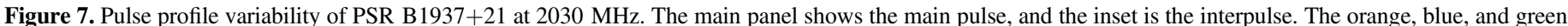

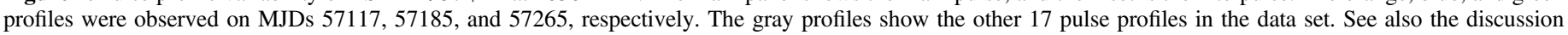
around polarization calibration in Section 5.6 and Figure 23. 


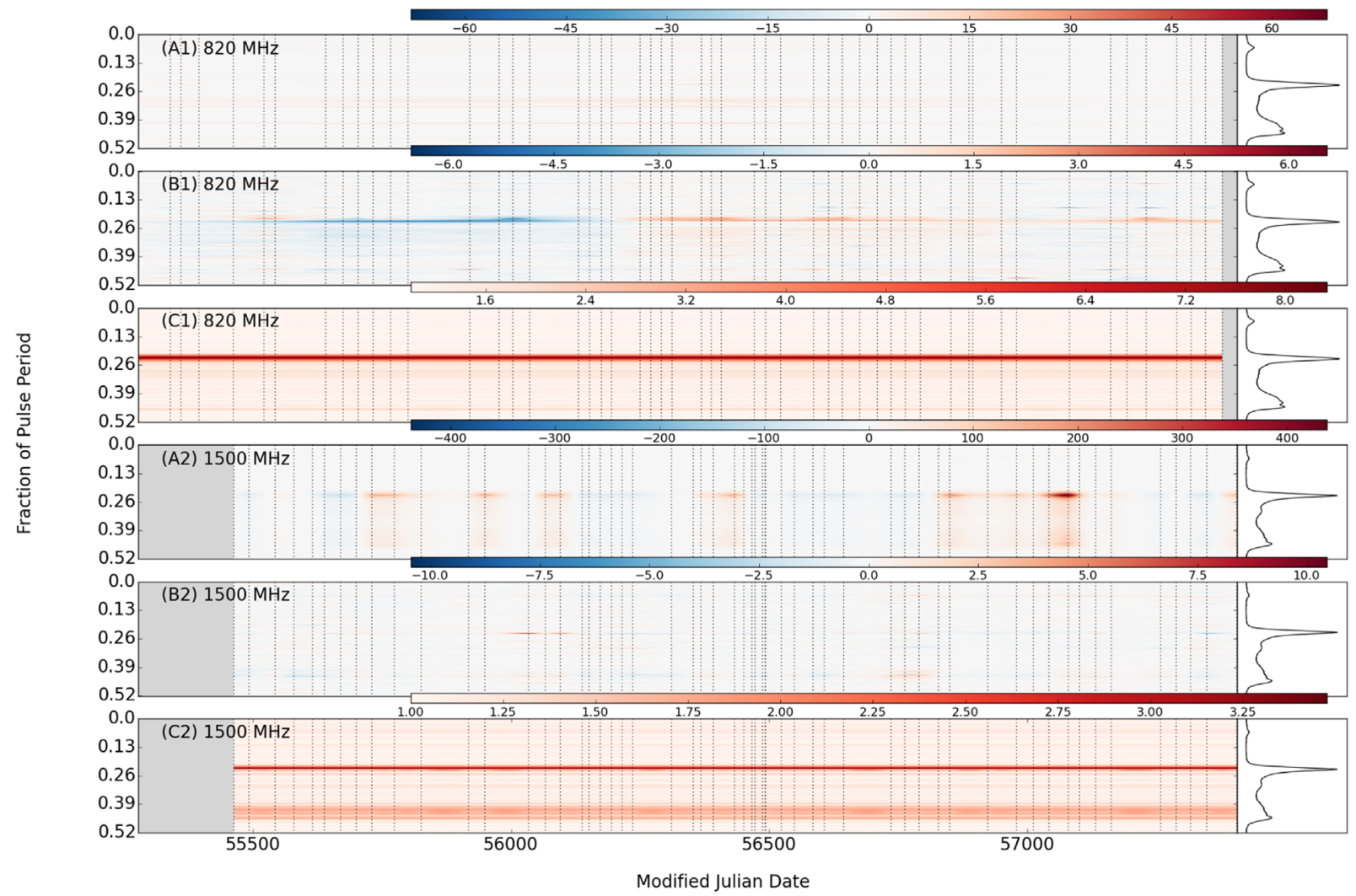

Figure 8. Variability maps for PSR J2145-0750. Otherwise, this figure is the same as Figure 3.

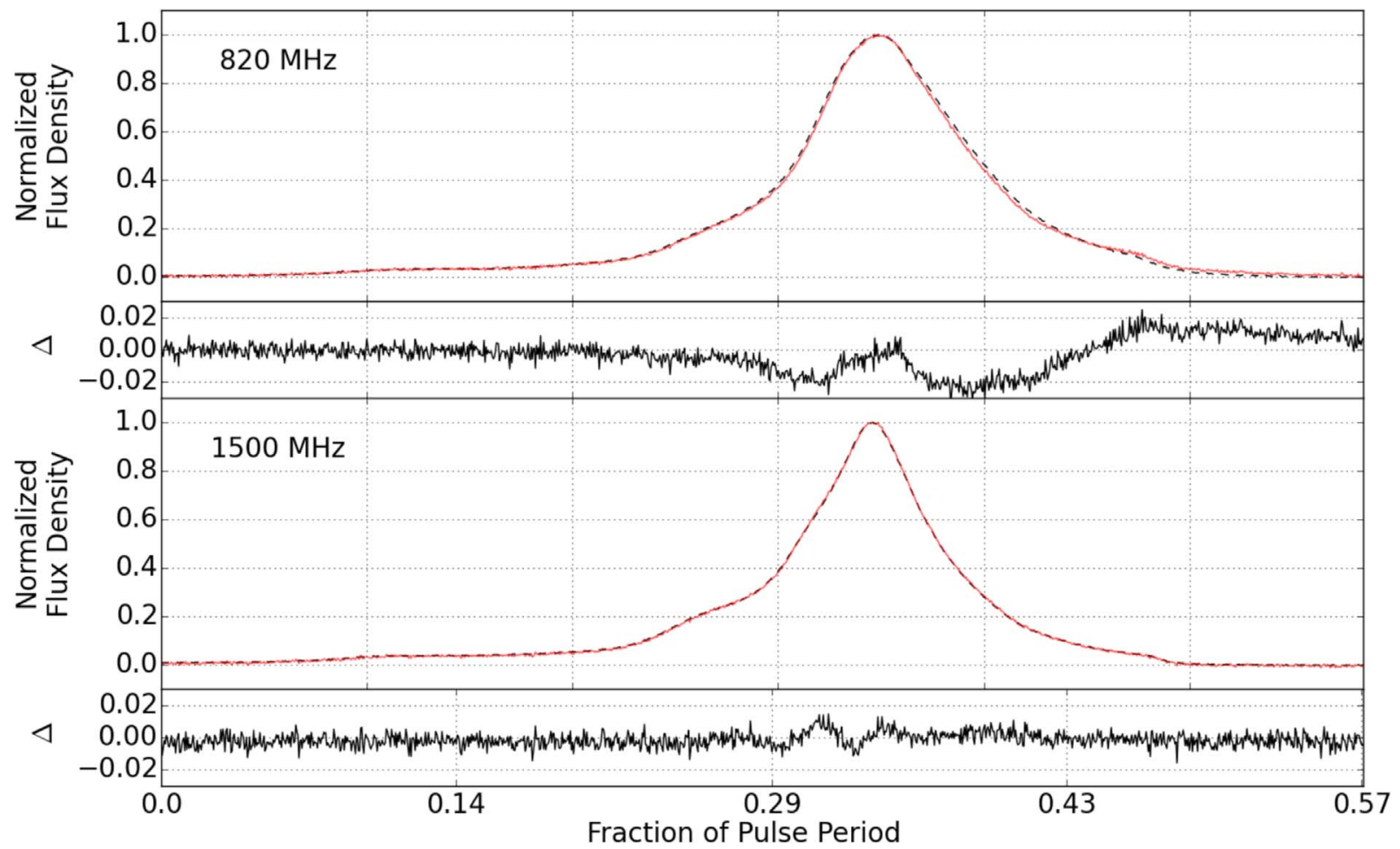

Figure 9. Combined pulse profiles of PSR J1643-1224 before (red) and after (black dashed) MJD 57074 (2015 February 21). The narrower panels show the red profile minus the black dotted profile $(\Delta)$. All profiles are normalized to the peak. 


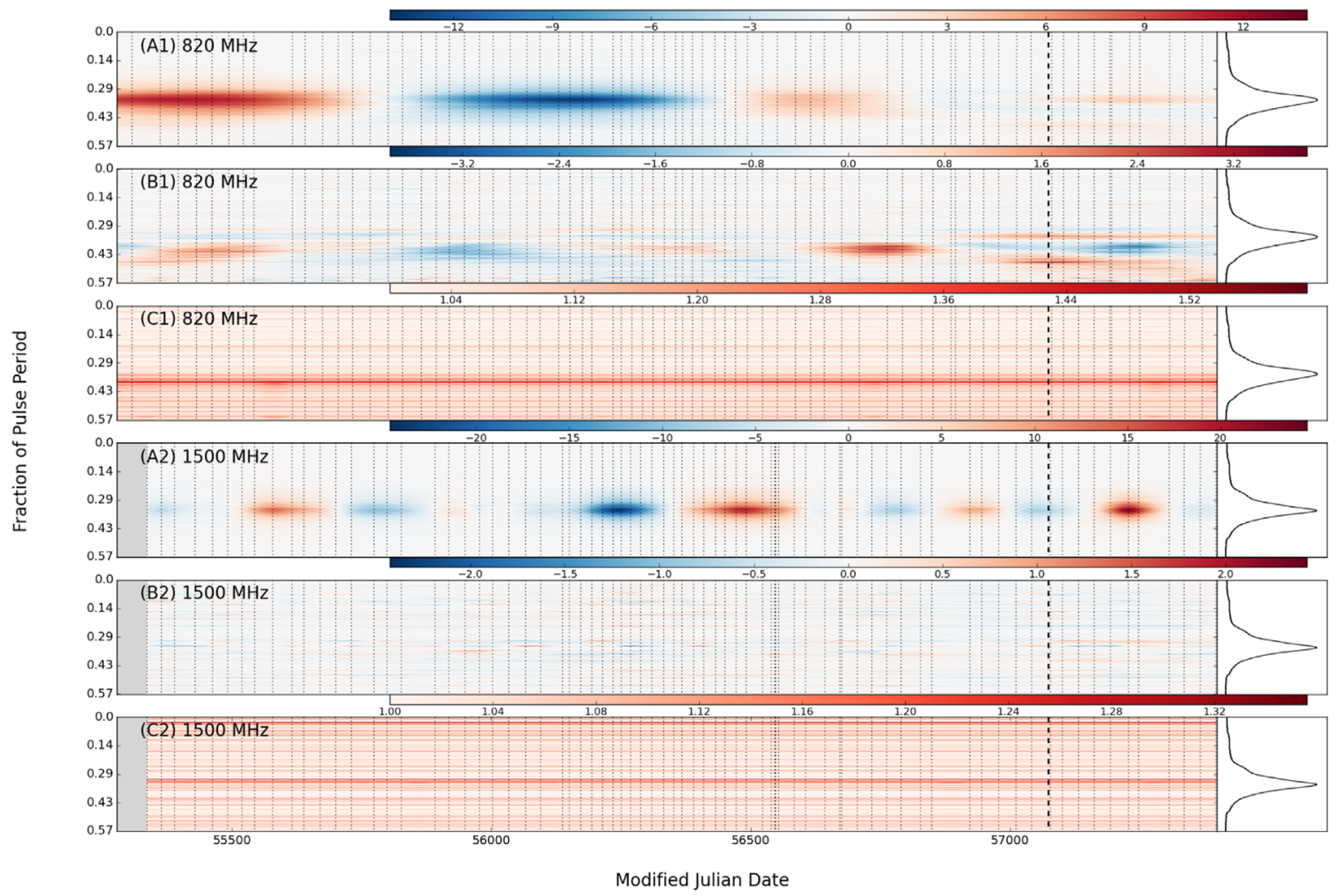

Figure 10. Variability maps for PSR J1643-1224. Otherwise, this figure is the same as Figure 3.

data, in which an emission feature moves away from the center of the pulse profile over a few hundred days, is more clearly shown in Figure 12.

\section{Discussion}

We have used a new profile alignment technique, GP regression, and multiple metrics to characterize the pulse profile evolution of 78 NANOGrav MSP data sets. All pulsars show flux density variations due to DISS and RISS. After flux density levels are normalized, the differences between the constant average model (for a particular data set) and the observed profiles for most of the pulsars is consistent with being due to additive white Gaussian noise; for the vast majority of pulsars, the mean standard deviation of their onpulse phase bins is less than a factor of two greater than that of their off-pulse bins. The three pulsars for which this factor is greatest are PSRs J1713+0747, B1937+21, and J2145-0750. Additionally, PSR J1643-1224 shows significant long-term variability, which has been previously identified.

\subsection{Profile and Timing Variability in J1643-1224}

As mentioned in Section 4.4, around 2015 February 21 (MJD 57074), Shannon et al. (2016) observe both TOA and pulse profile variations in PSR J1643-1224. The largest TOA perturbations are at $3100 \mathrm{MHz}$. They report a change that leaves permanent excess power in the leading edge of the $3100 \mathrm{MHz}$ and $1400 \mathrm{MHz}$ profiles. Their $700 \mathrm{MHz}$ observations show the least amount of timing variation around this date. TOA perturbations that begin around MJD 57074 can also be seen in NANOGrav data at both $820 \mathrm{MHz}$ and $1500 \mathrm{MHz}$ (Figure 11). As seen in column 3 of Table 1, however, the PSR J1643-1224 $1500 \mathrm{MHz}$ pulse profiles are the most stable of all the data sets analyzed in this work. This suggests that the pulse profile changes are not the cause of the TOA disruptions. At $820 \mathrm{MHz}$, we see more obvious pulse profile changes, but they occur across the whole data set, rather than abruptly around MJD 57074, as seen by Shannon et al. at $3100 \mathrm{MHz}$. The $820 \mathrm{MHz}$ changes seem to occur primarily at the trailing edge of the profile, which is, again, in contrast to the $3100 \mathrm{MHz}$ Parkes data, in which Shannon et al. see a significant excess signal in the leading edge after $\sim$ MJD 57074, occurring concurrently with a significant change in the timing residuals. At around the same time, the NANOGrav $820 \mathrm{MHz}$ GBT data show a profile feature drifting away from the central peak over a span of a few hundred days (Figure 12). A similar phenomenon occurred in the Crab pulsar in 1997 (Backer et al. 2000), which has been attributed to refraction and multiple imaging at the edge of a plasma cloud in the outer region of the Crab Nebula (Graham Smith et al. 2011). Only profiles at $3100 \mathrm{MHz}$ are shown in the Shannon et al. paper, and so a direct comparison of Parkes and GBT PSR J1643-1224 pulse profiles at similar frequencies around the time of the TOA disturbance has yet to been done.

Whenever considering the pulse phase at which profile variability occurs, it should be understood that different methods for alignment can show the variability to occur at different parts of the pulse profile.

\subsection{The Effect of Pulse Profile Shape Changes on TOAs}

A TOA is determined by a technique that matches the pulse profile from an individual observation, with a static pulse profile model (Taylor 1992; van Straten 2006). Any evolution of the pulse profile with time, therefore, will affect the TOA that is produced by this template matching procedure. As discussed in Section 1, in the NANOGrav 11-year data set, 


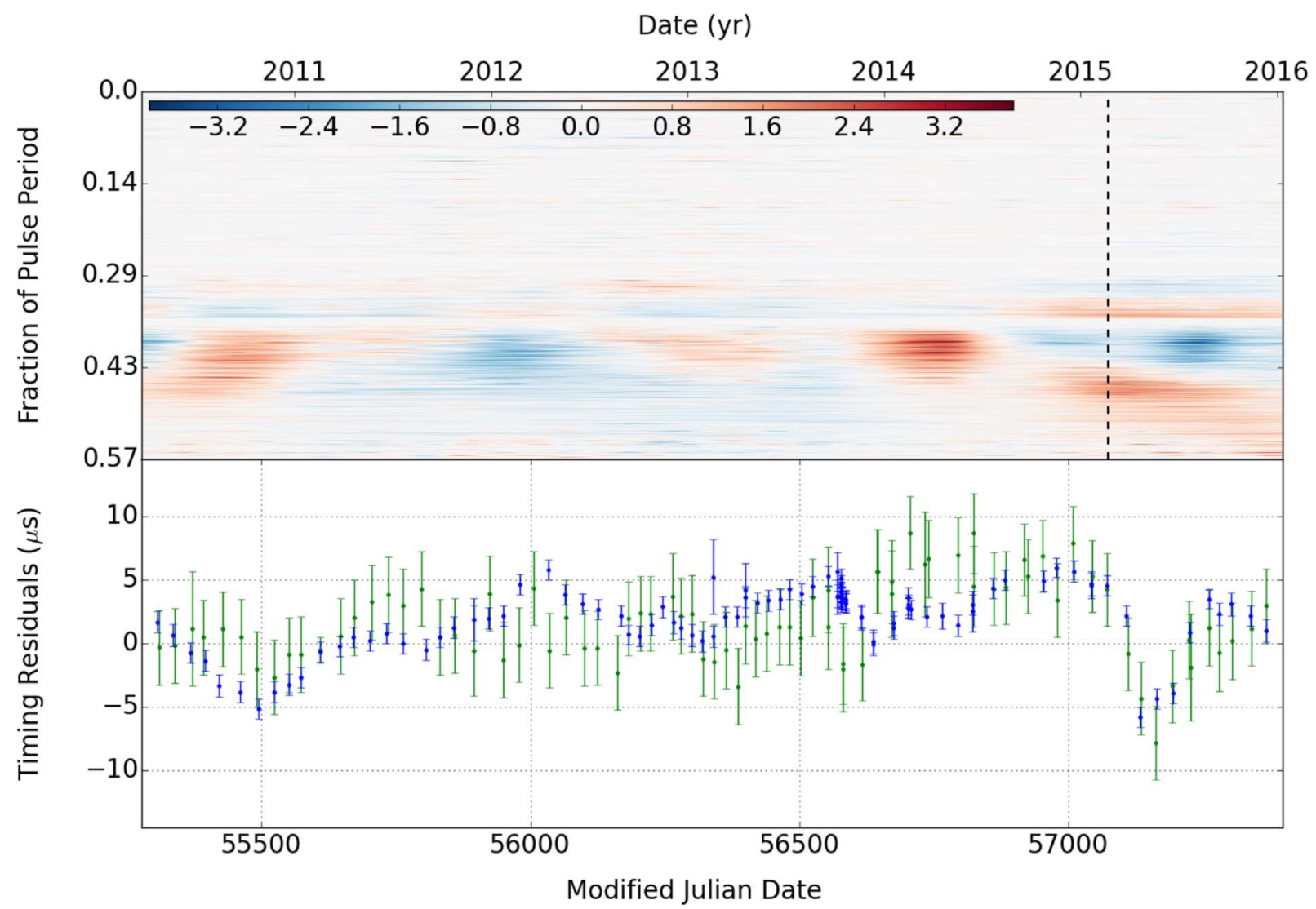

Figure 11. Profile and timing residuals of the J1643-1224 $820 \mathrm{MHz}$ data set observed at the GBT. The top panel is a variability map showing pulse profile shape changes after the observations have been normalized. Red regions indicate where the inferred pulse profile has an excess of flux density compared to the average for the data set. Blue indicates where it has a deficit. The unit for the variability map is the mean of the standard deviation of the off-pulse phase bins for the data set. This panel depicts the same data as Panel (B1) of Figure 10, with the addition of a solid vertical line, which denotes 2015 February 21 (MJD 57074), the date around which timing and profile changes were seen by Shannon et al. (2016). The bottom panel shows the TOA residuals for PSR J1643-1224 (Arzoumanian et al. 2018) at $820 \mathrm{MHz}$ (green) and $1500 \mathrm{MHz}$ (blue).

each frequency subband is used to produce TOAs via the template matching procedure. It should be stressed, therefore, that there are phenomena that could cause profile changes in the frequency-integrated pulse profiles while having little effect on the profiles of individual frequency channels and, therefore, on the NANOGrav TOAs.

In order to assess the magnitude of changes in TOA that result from the frequency-integrated pulse profile shape variability that we have seen, we employ the template matching procedure using the PYPULSE software package (Lam 2017). The fitPulse function performs the template matching procedure described in Taylor (1992); any two pulse profiles are cross-correlated in order to calculate a difference in TOA between them.

Before the template matching analysis was carried out, the relative alignment of the pulse profiles was performed. We have employed a new, objective pulse profile alignment technique that maximizes the number of pulse phase bins that are in agreement between profiles (see Section 3.1 for details). The nature of the alignment technique is such that we are insensitive to phase shifts caused by astrophysical processes such as timing noise. In this paradigm, the definition of the fiducial point (a reference point for timing measurements) becomes the phase at which the pulse profile has the least variability; without knowledge of the physical processes involved in the profile shape changes, we assert that this is a reasonable thing to do. In some cases, the profile shape change is quite dramatic and consequently has a dramatic effect on the
TOA, as calculated in Table 2. The mean and standard deviations in the table may be dominated by such outliers and be skewed as a consequence. The metric of $\langle|\Delta \mathrm{TOA}|\rangle$ and standard timing residuals are difficult to compare. We are not using traditional pulsar timing; we are instead essentially assuming that the pulsar is a perfect rotator and have defined the fiducial point as the most stable phase of the pulsar. Additionally, as discussed in Section 3.1, we are only aligning in single bin increments (with 2048 bin resolution), as it is sufficient for the profile profile variability analysis that is the focus of this work. Not aligning to fractions of a phase bin may also inflate the values in Table 2.

Table 2 shows the TOA changes $(\triangle T O A)$ induced by the pulse profile shape changes seen in PSRs J1643-1224, J1713 +0747 , B1937+21, and J2145-0750. The template model used is the average profile of all normalized observations that survived the analysis in Section 3. A value of $\triangle T O A$ was calculated for each observation. Template matching the average model with itself produces a $\triangle$ TOA value of zero by definition.

In the 12 data sets analyzed, the average value of the magnitude of $\triangle \mathrm{TOA}$ induced by the changing pulse profile shape is typically around three orders of magnitude larger than the average $1 \sigma$ uncertainty of the TOA measurements $\langle\sigma \mathrm{TOA}\rangle$.

In general, the potential causes of the pulse profile variability are scintillation, inaccurate DM, scatter broadening, instrumental and interference issues, jitter, or other emission changes intrinsic to the pulsar. We discuss each possibility in detail as follows. 


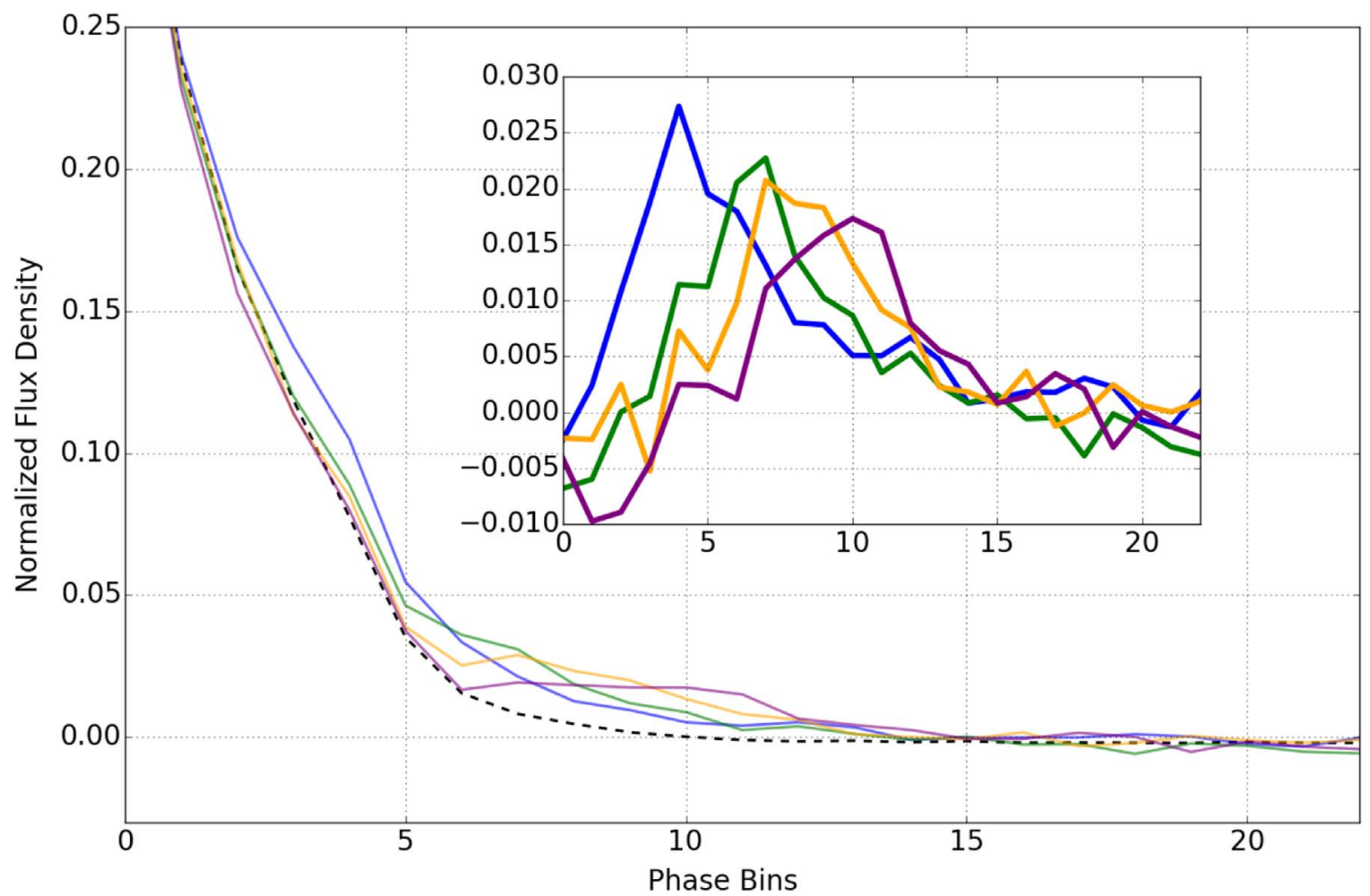

Figure 12. The main plot shows the trailing edge of the pulse profile for PSR J1643-1224 observed at $820 \mathrm{MHz}$. The 2048 phase bins that span the pulse period have been resampled to 64 in order to increase the profile S/N. The average profile for the data set is shown by a black dashed line. The four solid lines are the profiles as observed on MJDs 57102 (blue), 57307 (green), 57336 (orange), and 57369 (purple). The inset shows the difference between each colored profile in the main plot and the average pulse profile (observation minus average). The phase location of the maximum deviation can be seen to drift from left to right with time.

Table 2

The Changes in TOA Induced by Pulse Profile Variation for PSRs J1643-1224, J1713+0747, B1937+21, and J2145-0750

\begin{tabular}{|c|c|c|c|c|c|}
\hline Pulsar & Observing Frequency (MHz) & $\langle|\Delta \mathrm{TOA}|\rangle(\mu \mathrm{s})$ & $\sigma \Delta \mathrm{TOA}(\mu \mathrm{s})$ & Max. $\Delta$ TOA $(\mu \mathrm{s})$ & $\langle\sigma \mathrm{TOA}\rangle(\mathrm{ns})$ \\
\hline $\mathrm{J} 1643-1244$ & 820 & 0.75 & 0.93 & 5.00 & 3.44 \\
\hline J1643-1244 & 1500 & 0.390 .44 & 0.340 .40 & 1.651 .86 & 3.703 .52 \\
\hline $\mathrm{J} 1713+0747$ & 1400 & 1.37 & 2.36 & 12.81 & 2.50 \\
\hline $\mathrm{J} 1713+0747$ & 2030 & 1.27 & 1.45 & 7.40 & 4.90 \\
\hline $\mathrm{J} 1713+0747$ & 1500 & 0.890 .88 & 0.840 .84 & 4.943 .92 & 2.922 .90 \\
\hline $\mathrm{J} 1713+0747$ & 820 & 0.92 & 0.92 & 5.00 & 4.21 \\
\hline $\mathrm{B} 1937+21$ & 820 & 0.21 & 0.15 & 0.74 & 0.74 \\
\hline $\mathrm{B} 1937+21$ & 1500 & $0.23 \quad 0.17$ & $0.25 \quad 0.14$ & $2.08 \quad 0.58$ & 0.740 .64 \\
\hline $\mathrm{B} 1937+21$ & 1400 & 0.16 & 0.11 & 0.43 & 0.84 \\
\hline $\mathrm{B} 1937+21$ & 2030 & 0.22 & 0.12 & 0.48 & 1.88 \\
\hline $\mathrm{J} 2145-0750$ & 820 & 3.26 & 2.61 & 10.10 & 15.26 \\
\hline $\mathrm{J} 2145-0750$ & 1500 & 1.961 .92 & 1.331 .22 & $5.42 \quad 4.12$ & $15.18 \quad 16.10$ \\
\hline
\end{tabular}

Note. $\langle|\Delta \mathrm{TOA}|\rangle$ is the mean of the absolute TOA change induced by the pulse profile shape changes. $\sigma \Delta \mathrm{TOA}$ is the standard deviation of the $\Delta \mathrm{TOA}$ distribution. Max. $\triangle \mathrm{TOA}$ is the largest TOA changed induced in the data set, and $\langle\sigma \mathrm{TOA}\rangle$ is the mean uncertainty in the TOA calculations. The data sets observed at $1500 \mathrm{MHz}$ have two values for each variability metric. The left of the pair relates to profiles that were polarization calibrated only by a local noise diode, and the right to profiles that were additionally polarization calibrated using the full Mueller matrix (see Section 3).

\subsection{Diffractive Interstellar Scintillation (DISS)}

DISS is the frequency-dependent modulation of pulsar flux density. If a pulse profile is a composite of a wide range of equally weighted frequency channels (as it is in this analysis), scintillation will necessarily lead to pulse profile changes, providing that (i) the profile evolves with frequency across the observing band, (ii) the scintillation bandwidth is not much smaller than the observing bandwidth, and (iii) the scintillation timescale is not much smaller than the timescale of the observation. In each data set for PSRs J1643-1224, J1713 +0747 , B1937+21, and $\mathbf{J} 2145-0750$, scintillation is occurring to differing degrees, affecting the relative flux in different parts of the observing band (see Figure 13). There is also some pulse profile shape evolution across the observing band for PSRs J1713+0747, B1937+21, and J2145-0750. Therefore, scintillation plays at least some part in the pulse profile variability seen in the analysis of these three pulsars. Relatively little pulse profile shape evolution is seen across the observing band for PSR J1643-1224.

Levin et al. (2016) showed that the average scintillation bandwidth for PSR B1937+21 at $1500 \mathrm{MHz}$ is around $2.8 \mathrm{MHz}$, which is close to the resolution limit. Keith et al. (2013) give a 


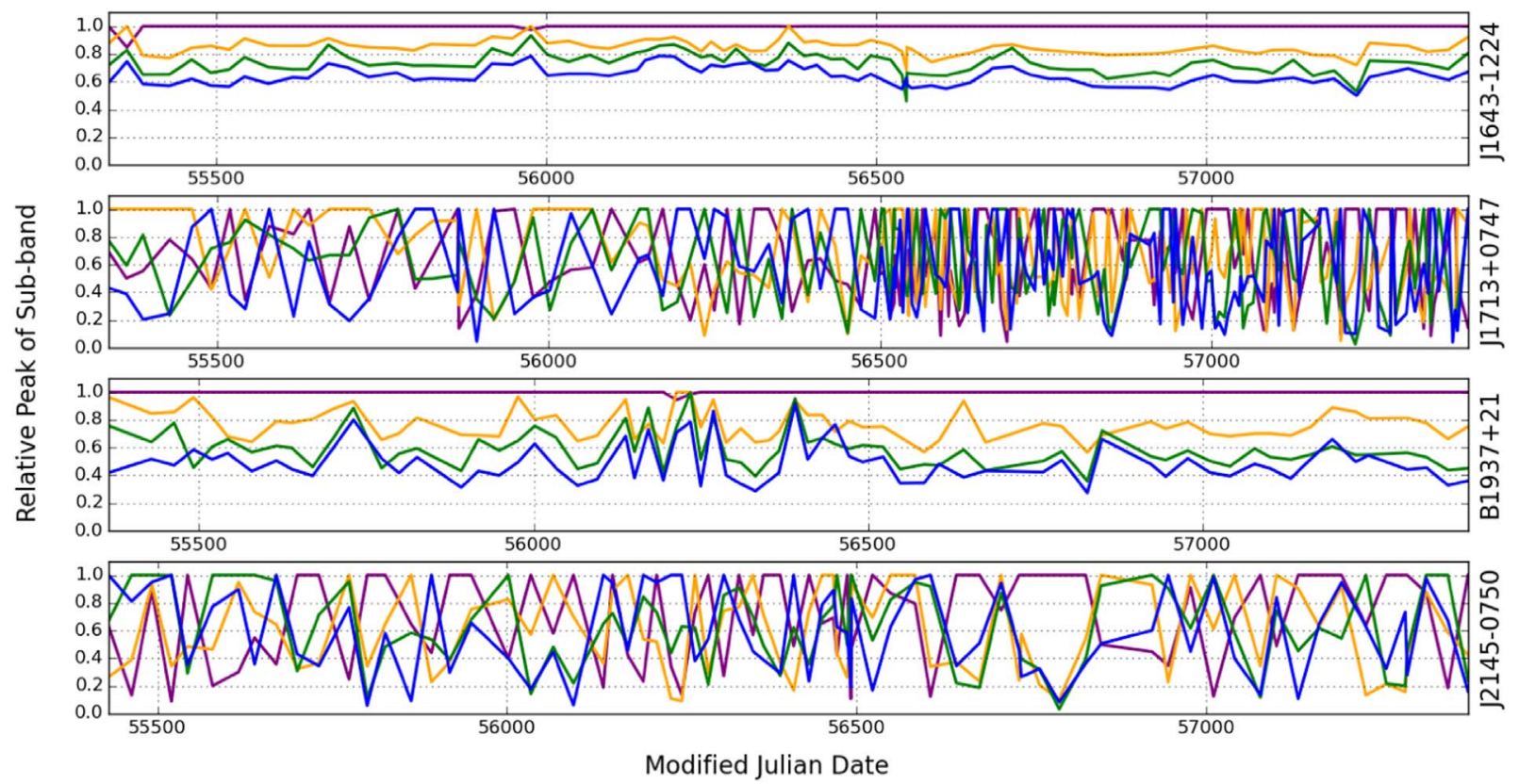

Figure 13. Relative brightness of frequency subbands within the observing band at $1500 \mathrm{MHz}$. The $\sim 800 \mathrm{MHz}$ bandwidths are divided into four $\sim 200 \mathrm{MHz}$ bandwidths. From lowest to highest frequency, the subbands are represented by purple, orange, green, and blue. From top to bottom, the panels show the relative subband brightness for PSRs J1643-1224, J1713+0747, B1937+21, and J2145-0750.

value of $1.2 \mathrm{MHz}$ at a reference frequency of $1500 \mathrm{MHz}$. The fractional uncertainty in the scintillation bandwidth is $1 / \sqrt{N_{\text {iss }}}$, where

$$
N_{\mathrm{iss}} \approx\left(1+\zeta \frac{\Delta \nu}{\Delta \nu_{d}}\right)\left(1+\zeta \frac{T}{\Delta t_{d}}\right)
$$

and where $\zeta$ is an empirically determined coefficient $(\zeta \approx$ $0.1-0.2), \Delta \nu$ is the receiver bandwidth, $\Delta \nu_{d}$ is the scintillation bandwidth, $T$ is the integration time of the observation, and $\Delta t_{d}$ is the scintillation timescale (Cordes et al. 1990). Using $\Delta \nu_{d}=1.2 \mathrm{MHz}$ and $\Delta t_{d}=327 \mathrm{~s}$ from Keith et al. (2013) and $\zeta=0.2$, the fractional uncertainty in scintillation bandwidth for a 30 minute, $800 \mathrm{MHz}$ bandwidth observation is $\sim 6 \%$. For the 1400 and $2030 \mathrm{MHz}$ centered observations in this analysis, the observing bandwidth is $800 \mathrm{MHz}$; for $1500 \mathrm{MHz}$, it is $700 \mathrm{MHz}$; and for the $820 \mathrm{MHz}$ centered observations, it is $200 \mathrm{MHz}$. As these bandwidths are so much larger than the scintillation bandwidths for PSR B1937+21, we expect to see scintillation effects largely averaging out across the observing band. Figure 13 shows that although the scintillation observed in PSR B1937+21 is much less than that observed in PSRs J1713+0747 and J2145-0750, the relative weighting of different parts of the observing band does change with time.

Levin et al. were unable to calculate the scintillation bandwidth for PSR J1643-1224, limited by the frequency resolution of their observations. Keith et al. (2013) give a value of $22 \mathrm{kHz}$ at a reference frequency of $1500 \mathrm{MHz}$. Using Equation (2), setting $\Delta \nu_{d}$ to $22 \mathrm{kHz}$ and $\Delta t_{d}$ to $582 \mathrm{~s}$ from Keith et al. and setting $\zeta$ to 0.2 gives a scintillation bandwidth fractional uncertainty for a 30 minute, $800 \mathrm{MHz}$ bandwidth observation of $\sim 1 \%$. Again, these observing bandwidths are so much larger than the scintillation bandwidth that we expect to see scintillation effects averaging out across the observing band. Despite this, the top panel of Figure 13 indicates that scintillation is causing some changes in the relative flux density across the observing band for PSR J1643-1224.

The average scintillation bandwidth reported at $1500 \mathrm{MHz}$ by Levin et al. is $21.1 \mathrm{MHz}$ for PSR $\mathrm{J} 1713+0747$ and $47.8 \mathrm{MHz}$ for PSR J2145-0750. The second to top and bottom panels in Figure 13 indicate clear scintillation for PSRs J1713+0747 and J2145-0750, respectively.

Figure 14 illustrates the nature of typical pulse profile variations that we see in the PSR J2145-0750 data set at $820 \mathrm{MHz}$. The figure shows that when divided into four $\sim 50 \mathrm{MHz}$ frequency bands, the pulse profile shapes of the subbands are largely stable between MJDs 55361 and 56792 (see Panels (B2) and (C2)) and the relative flux densities are not (see Panels (B1) and (C1)). Between these two observation dates, the relative weighting of parts of the observing band has been changed by scintillation. As the different parts of the band have different profile shapes, a modification of the frequencyintegrated pulse profile necessarily results. The pulse profile changes that are seen in PSR J2145-0750 are, therefore, consistent with the effects of scintillation.

For PSRs J1643-1224, J1713+0747, and B1937+21, some variability is not consistent with the effects of scintillation. In Figure 15, the pulse profile variations centered at $820 \mathrm{MHz}$ are seen to correlate across the observing band for both PSR J1643 -1224 and PSR B1937+21. We do not expect such effects to be the result of scintillation. Figure 16 shows that the systematic pulse profile variability of PSR J1713+0747 at $1500 \mathrm{MHz}$ is seen in only two of the four frequency subbands.

Furthermore, for some observations, we see that the evolution of the pulse profile across the observing band is different than for others. An example in PSR J1713+0747 can be seen by comparing panels (B2) and (C2) in Figure 17. It is not clear how such differences could be caused by scintillation. 

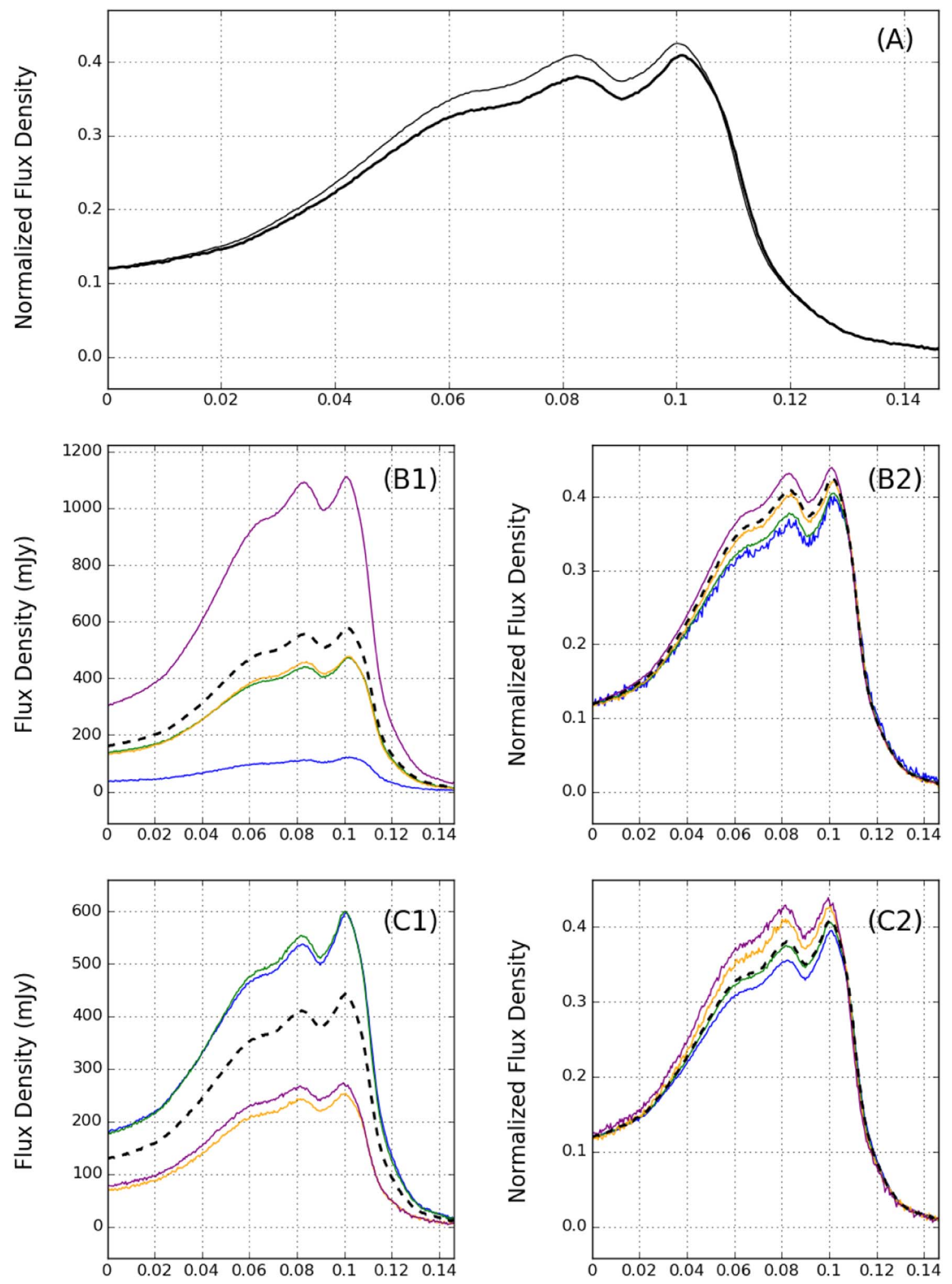

\section{Fraction of Pulse Period}

Figure 14. Two contrasting pulse profiles of PSR J2145-0750 at $820 \mathrm{MHz}$. Panel (A) shows two pulse profiles aligned and normalized by their peaks. The peak is not shown; all panels focus only on a subsection of the profile. This is done to allow shape changes to be seen clearly. The thin line is the pulse profile as observed on MJD 55361 and the thick line on MJD 56792. Panel (B1) shows the MJD 55361 observation split into four frequency subbands, each spanning 50 MHz. From lowest to highest frequency, the subbands are represented by purple, orange, green, and blue profiles. The black dashed line shows the frequency-integrated profile. In Panel (B2), the subband and frequency-integrated profiles have also been normalized to, and aligned by, the peak. Panels (C1) and (C2) show the same as (B1) and (B2), respectively, but for the observation made on MJD 56792 

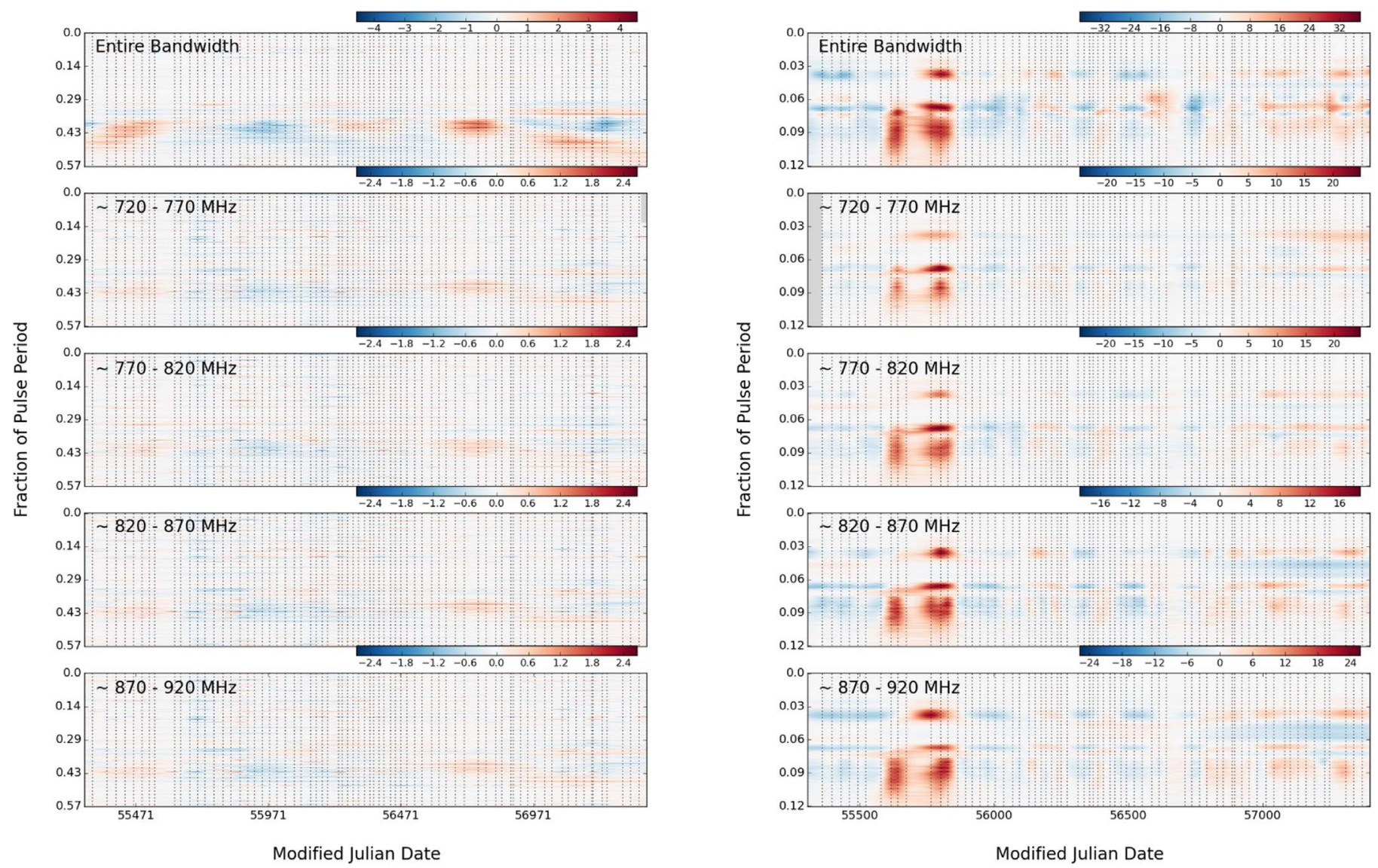

Figure 15. Variability maps for GUPPI data sets broken down into subbands of observational frequency. The left panel panel shows observations of PSR J1643-1224 with a central frequency of $\sim 820 \mathrm{MHz}$. The right panel shows the same for PSR B1937+21. The unit for all panels is the mean of the standard deviation of the offpulse phase bins for the relevant subband data set. Otherwise, this figure is the same as Figure 3.

\subsection{Inaccurate DM}

As an electromagnetic signal travels though the IISM, its interaction with free electrons produces a frequency-dependent time delay that scales as $\nu^{-2}$, where $\nu$ is the signal frequency. The magnitude of this delay is proportional to the integrated column density of electrons along the path of the signal, which is known as the DM. If we fail to correct for such frequencydependent time delays, an integrated pulse profile that is created by summing a signal detected across a range of observing frequencies will necessarily appear smeared out when compared to the intrinsic pulse shape. Although correcting for such signal dispersion is routine, DMs are well known to vary with epoch both systematically and stochastically (Keith et al. 2013; Lam et al. 2016a; Jones et al. 2017), due primarily to a changing line of sight. NANOGrav measures the value of DM at nearly every observing epoch (Arzoumanian et al. 2015), but an inaccurate DM value can lead to a modified pulse profile.

Because NANOGrav calculates TOAs for all frequency channels, a further complication is added to the determination of DM. Pulse shapes vary with frequency, but only a single standard template is used in the template matching procedure. This produces small systematic frequency-dependent perturbation in the TOAs, in addition to the $\nu^{-2}$ offsets due to dispersion. To compensate for this, an additional timing delay is added to all timing models, where

$$
\Delta t_{\mathrm{FD}}=\sum c_{i} \log \left(\frac{\nu}{1 \mathrm{GHz}}\right)^{i}
$$

and the coefficients $c_{i}$ are fit parameters in the timing model (Arzoumanian et al. 2015). When finding the best-fit timing model parameters for a pulsar, DM and $\Delta t_{\mathrm{FD}}$ are somewhat covariant, and so the best-fit DM value can change significantly, dependent on whether $\Delta t_{\mathrm{FD}}$ is included in the timing model.

For the purposes of creating the frequency-integrated pulse profiles employed in this variability analysis, we have calculated the best-fit DM parameters without the inclusion of the $\Delta t_{\mathrm{FD}}$ parameters that are necessary for TOA determination in individual frequency channels. This minimizes smearing when generating the frequency-integrated pulse profiles.

Jones et al. (2017) report that PSR J1713+0747 has a DM of $\sim 16 \mathrm{pc} \mathrm{cm}^{-3}$, which is typically seen to vary on the order of $10^{-4} \mathrm{pc} \mathrm{cm}^{-3}$ on approximately yearly timescales. A $1400 \mathrm{MHz}$ observation that has a DM inaccuracy of a few $10^{-4} \mathrm{pc} \mathrm{cm}^{-3}$ would only introduce a delay across an $800 \mathrm{MHz}$ bandwidth of a few tenths of a microsecond. A single phase bin in our analysis of PSR J1713+0747 covers an order of magnitude more time than this $(2.23 \mu \mathrm{s})$. Figure 18 demonstrates that to produce some of the most modified pulse profiles in the $1400 \mathrm{MHz}$ data set, the DM would have to be incorrect by the order of $10^{-2} \mathrm{pc} \mathrm{cm}^{-3}$, which is around a hundred times larger than the DM variations that we observe for this pulsar.

PSR B1937+21 is calculated to have a DM of $\sim 71 \mathrm{pc} \mathrm{cm}^{-3}$, which is typically seen to vary on the order of $10^{-3} \mathrm{pc} \mathrm{cm}^{-3}$ on approximately yearly timescales. A $1400 \mathrm{MHz}$ observation that has a DM inaccuracy of a few $10^{-3} \mathrm{pc} \mathrm{cm}^{-3}$ would introduce a 


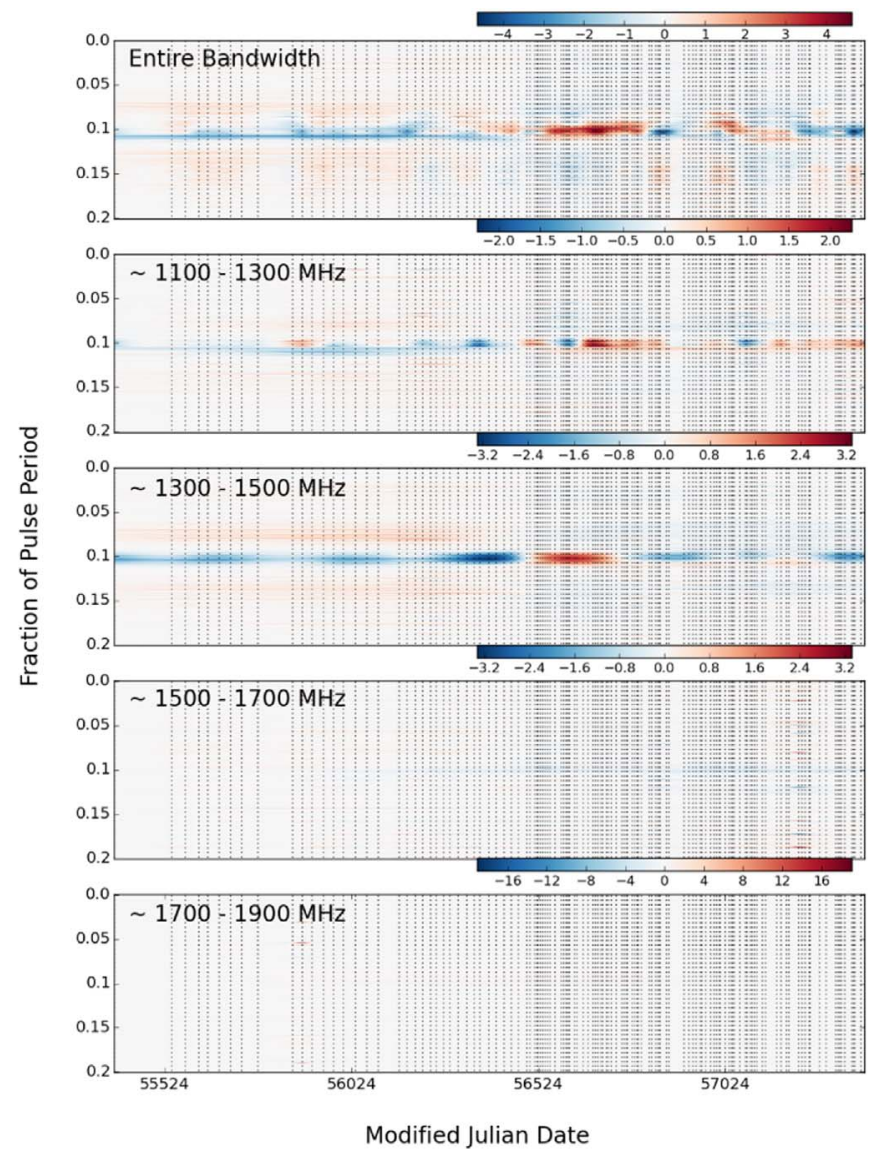

Figure 16. Variability maps for PSR J1713+0747 GUPPI observations at a central frequency of $\sim 1500 \mathrm{MHz}$, broken down into subbands of observational frequency. The unit for all panels is the mean of the standard deviation of the off-pulse phase bins for the relevant subband data set. Otherwise, this figure is the same as Figure 3.

delay across an $800 \mathrm{MHz}$ bandwidth of a few microseconds. This is the equivalent of a few PSR B $1937+21$ phase bins (each spanning $0.76 \mu \mathrm{s}$ ). Figure 19 shows that to produce some of the profile variations seen in the $820 \mathrm{MHz}$ data set, the DM would have to change by around $10^{-3}-10^{-2} \mathrm{pc} \mathrm{cm}^{-3}$, which is comparable to the typical DM fluctuations seen in this pulsar.

A DM of $62.4 \mathrm{pc} \mathrm{cm}^{-3}$ with approximately yearly fluctuations of around $10^{-3} \mathrm{pc} \mathrm{cm}^{-3}$ is reported for PSR J1643-1224 by Jones et al.; an incorrect DM value of this magnitude would introduce a delay at $820 \mathrm{MHz}$ across an $200 \mathrm{MHz}$ bandwidth of a few microseconds. This is the equivalent of one or two PSR J1643-1224 phase bins (each spanning 2.26 $\mu$ s). However, the phase drifts that are seen in Figure 12 are not suggestive of profile changes induced by incorrect DM measurements, as the modifications in each observation are localized in relatively narrow regions of pulse phase; a more smeared effect would be expected from incorrect DM values.

PSR J2145-0750 has a DM of $9 \mathrm{pc} \mathrm{cm}^{-3}$ with typical variations on the order of $10^{-3} \mathrm{pc} \mathrm{cm}^{-3}$ occurring on approximately yearly timescales, as reported by Jones et al.; inaccuracies on this scale would introduce a delay of a few microseconds across the $200 \mathrm{MHz}$ bandwidth at $820 \mathrm{MHz}$. This is only a fraction of a J2145-0750 phase bin, which spans $\sim 8 \mu \mathrm{s}$. Additionally, even in the most deviant pulse profiles in the data set, some sharp features remain, which would be smeared out when a DM inaccuracy (of the magnitude needed to replicate the profile changes) exists.
Based on these calculations, an inaccurate (but realistic) DM value used to dedisperse the pulsar signal when producing a frequency-integrated pulse profile could produce shape changes in PSRs B1937+21 and J1643-1224, but is unlikely to produce those observed in PSR J1713+0747 or J2145-0750.

\subsection{Temporal Broadening from Scattering}

Electromagnetic waves traveling through the IISM are scattered and follow different paths to the observer. This can, therefore, lead to the broadening of an observed pulse profile; an intrinsically narrow pulse will broaden due to scattering, producing an exponential decay of the pulse with a characteristic timescale $\tau$ known as the scattering timescale. Scatter broadening is a frequency-dependent effect, with $\tau \propto \nu^{-4.4}$ for a thin screen scattering model (Cordes \& Lazio 1991).

Levin et al. (2016) determine average scattering timescales via the measurement of scintillation bandwidths $\Delta \nu$ in the dynamic spectra of the observations, using the relationship

$$
2 \pi \Delta \nu \tau \sim 1
$$

(Cordes \& Rickett 1998). For PSR B1937+21, Levin et al. calculate that at $1500 \mathrm{MHz}$, the average $\tau$ is around $44 \mathrm{~ns}$. This is close to the limit imposed by the frequency resolution of their observations. For some epochs, therefore, no scintillation bandwidth could be measured, meaning only a lower limit for $\tau$ on the order of tens of nanoseconds could be inferred. At $820 \mathrm{MHz}$, these values translate to scattering timescales on the order of a microsecond or more. Cordes et al. (1990) measure the scattering timescale at $430 \mathrm{MHz}$ for the main pulse of PSR B $1937+21$ to be $25 \pm 2 \mu \mathrm{s}$ and $30 \pm 2 \mu \mathrm{s}$ for the interpulse. Assuming a thin screen scattering model, this translates to a $\tau$ value of a few microseconds at $820 \mathrm{MHz}$. At $327 \mathrm{MHz}$, Ramachandran et al. (2006) measure $120 \mu$ s with an rms variation of $20 \mu \mathrm{s}$. With the thin screen scattering assumption, $120 \mu \mathrm{s}$ at $327 \mathrm{MHz}$ translates to approximately $3 \mu \mathrm{s}$ at $820 \mathrm{MHz}$. Keith et al. (2013) give a scintillation bandwidth of 1.2 MHz for PSR B1937+21, which translates to a scattering timescale of approximately $1.8 \mu$ s at $820 \mathrm{MHz}$. We simulate the effects of thin screen scatter broadening by convolving a pulse profile with a one-sided exponentially decaying function. We use a one-sided exponential function as an approximation to the pulse broadening function caused by interstellar scattering. Actual pulse broadening functions are more rounded at the origin due to the finite thickness of a scattering screen and can have more slowly decaying tails if there is a wide range of scattering length scales, as with a Kolmogorov medium. In Figure 20, such a simulation of scatter broadening for PSR B1937+21 shows that if the nature and magnitude of the pulse profile shape changes we see in the $820 \mathrm{MHz}$ data set were produced by thin screen scatter broadening, then $\tau$ would have to be on the order of microseconds, which is consistent with the findings of Levin et al., Cordes et al., Ramachandran et al., and Keith et al. This translates to a scintillation bandwidth less than $1 \mathrm{MHz}$. Additionally, the strongly correlated variability seen between the main pulse and the interpulse of PSR B1937+21 (most clearly illustrated at $820 \mathrm{MHz}$ in Panel (B1) of Figures 5 and 6) is consistent with what would be expected from a scatter 

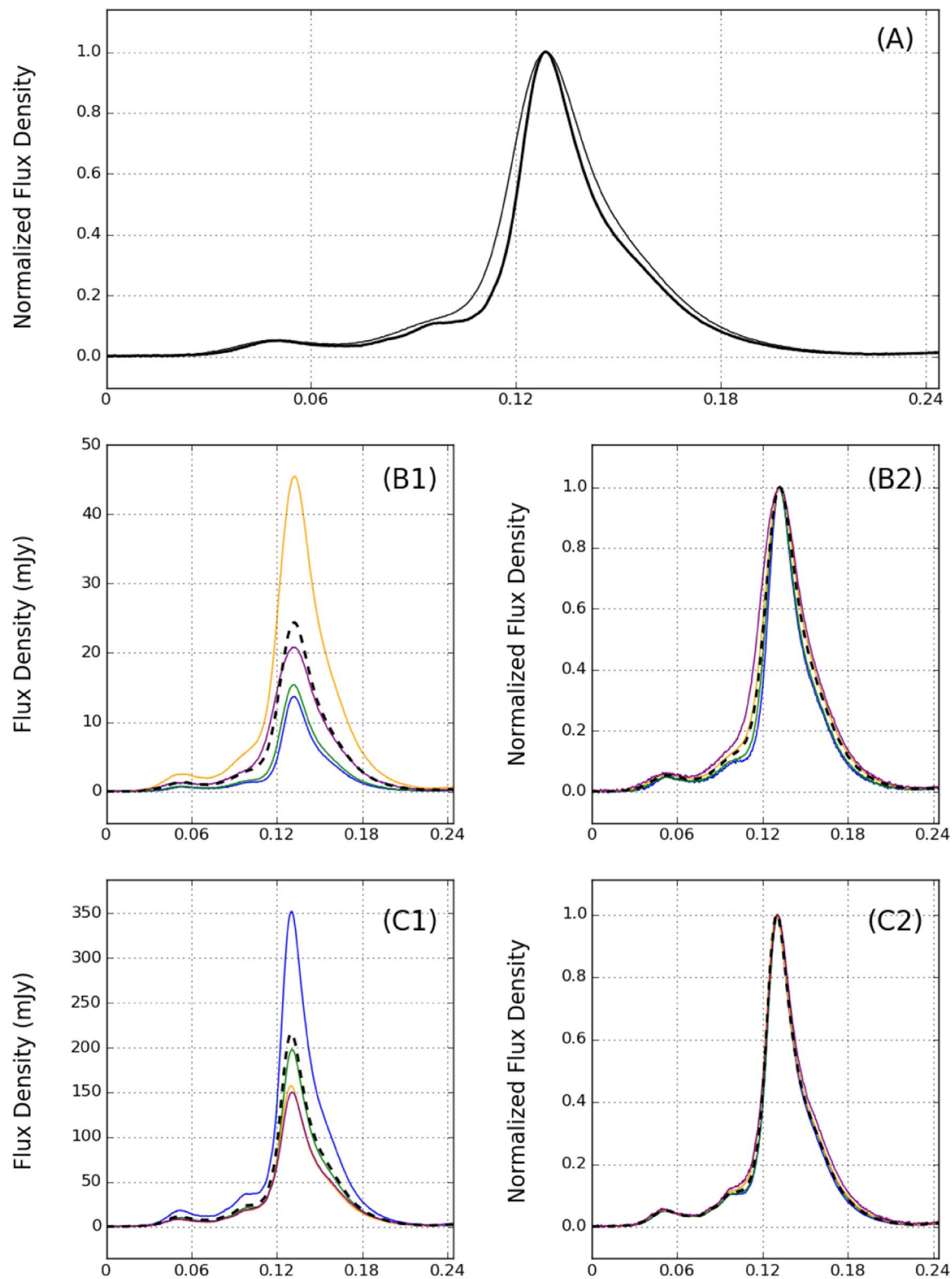

Fraction of Pulse Period

Figure 17. Two contrasting pulse profiles of PSR J1713+0747 at $1400 \mathrm{MHz}$. Panel (A) shows two pulse profiles aligned and normalized by their peaks. This is done to allow shape changes to be seen clearly. The thin line is the pulse profile as observed on MJD 56360, and the thick line is the pulse profile as observed on MJD 57076. Panel (B1) shows the MJD 56360 observation split into four frequency subbands, each spanning $200 \mathrm{MHz}$. From lowest to highest frequency, the subbands are represented by purple, orange, green, and blue profiles. The black dotted line shows the frequency-integrated profile. In Panel (B2), the subband and frequencyintegrated profiles have also been normalized to and aligned by the peak. Panel (C1) and (C2) show the same as (B1) and (B2) respectively, but for the observation made on MJD 57076. We see from Panels (B2) and (C2) that the evolution of the pulse profile across the observing band is different for the two observations. 


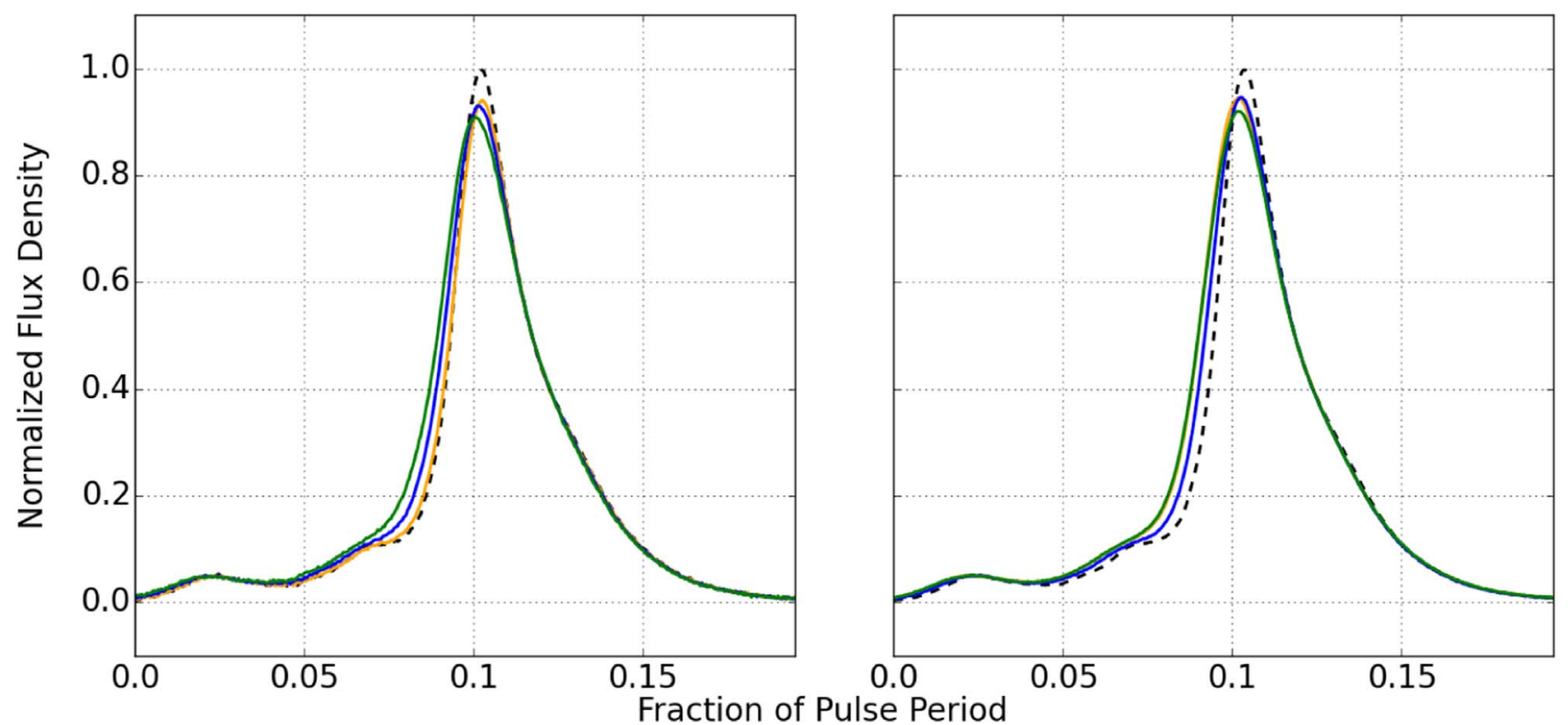

Figure 18. How the frequency-integrated pulse profiles of PSR J1713+0747 change with DM at $1400 \mathrm{MHz}$. Left panel: the black dashed profile was dedispersed at a $\mathrm{DM}$ of $15.990 \mathrm{pc} \mathrm{cm}^{-3}$. The orange, blue, and green profiles were dedispersed with $0.04,0.06$, and $0.08 \mathrm{pc} \mathrm{cm}^{-3}$ added, respectively. Right panel: three observations in the data set showing large deviations from the average pulse profile. The black dashed profile is the average for the data set. The orange, blue, and green profiles were recorded on MJDs 56360, 56598, and 57239, respectively.

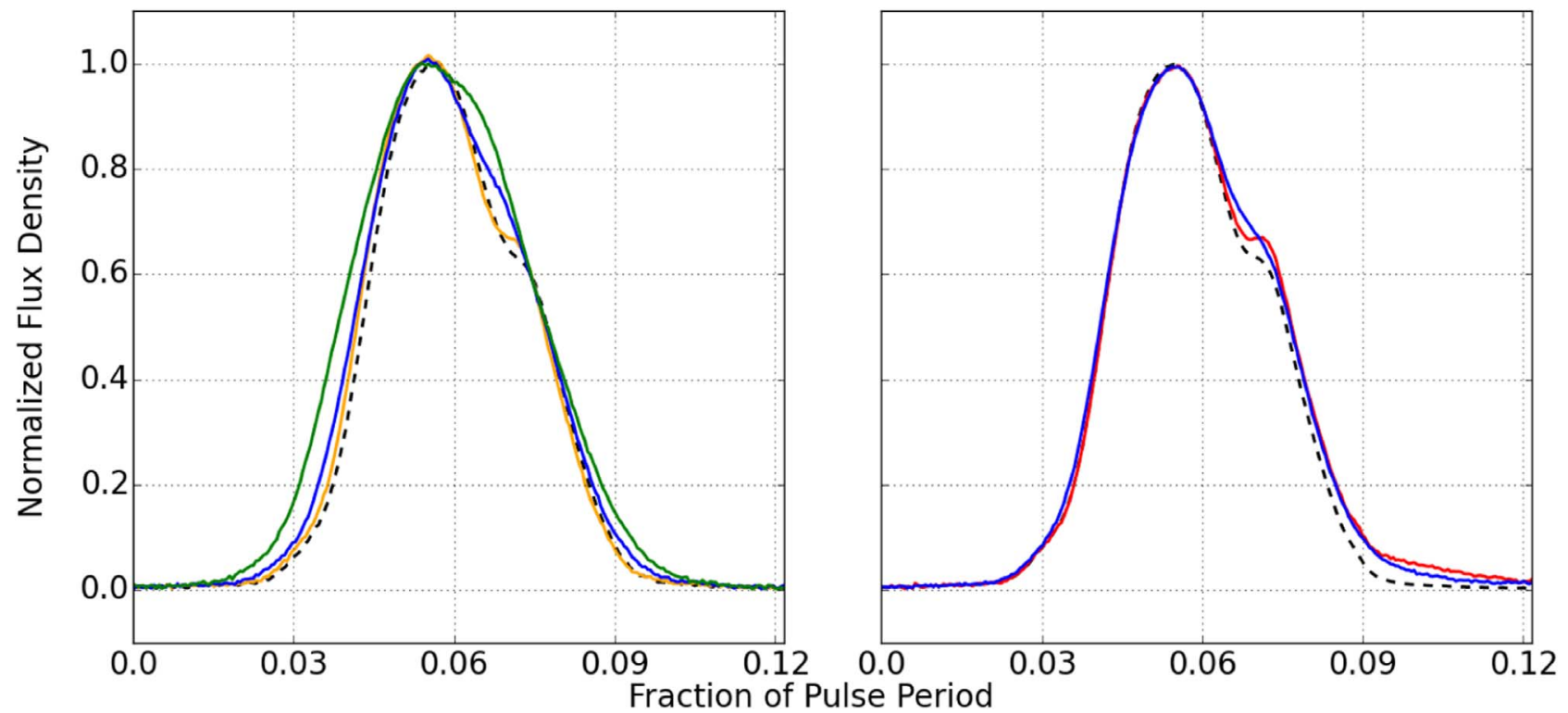

Figure 19. How the frequency-integrated pulse profiles of PSR B1937+21 change with DM at $820 \mathrm{MHz}$. Left panel: the black dashed profile was dedispersed at a $\mathrm{DM}$ of $71.025 \mathrm{pc} \mathrm{cm}^{-3}$. The orange, blue, and green profiles were dedispersed with $0.005,0.01$, and $0.015 \mathrm{pc} \mathrm{cm}^{-3}$ subtracted, respectively. Right panel: two observations in the data set showing large deviations from the average pulse profile. The black dashed profile is the average for the data set. The red profile was recorded on MJD 55641, and the blue profile was recorded on MJD 55765.

broadened signal (or one modified by propagation effects in general). However, a similar effect could also result from global changes in the pulsar magnetosphere, and so intrinsic variability cannot be ruled out on this basis.

For PSRs J1713+0747 and J2145-0750, Levin et al. (2016) find that $\tau$ is on the order of ns at $1500 \mathrm{MHz}$. These scattering timescales are much too small for scatter broadening to significantly affect the pulse profile shapes.

The nature of the pulse profile shape changes observed in PSR J1643-1224 cannot be well replicated simply by the convolution of a one-sided decaying exponential function; the phase range over which the profile is modified is usually relatively narrow and is also seen to drift with time. However, IISM structure that is close to the line of sight could permit such transient profile components via the deflection of radio waves back to the observer. Such behavior has been seen previously in other pulsars (Backer et al. 2000; Michilli et al. 2018).

\subsection{Instrumental Issues and RFI}

The pulse profile shape changes of PSR J1713+0747 at $2030 \mathrm{MHz}$ seem to mainly occur approximately between the 


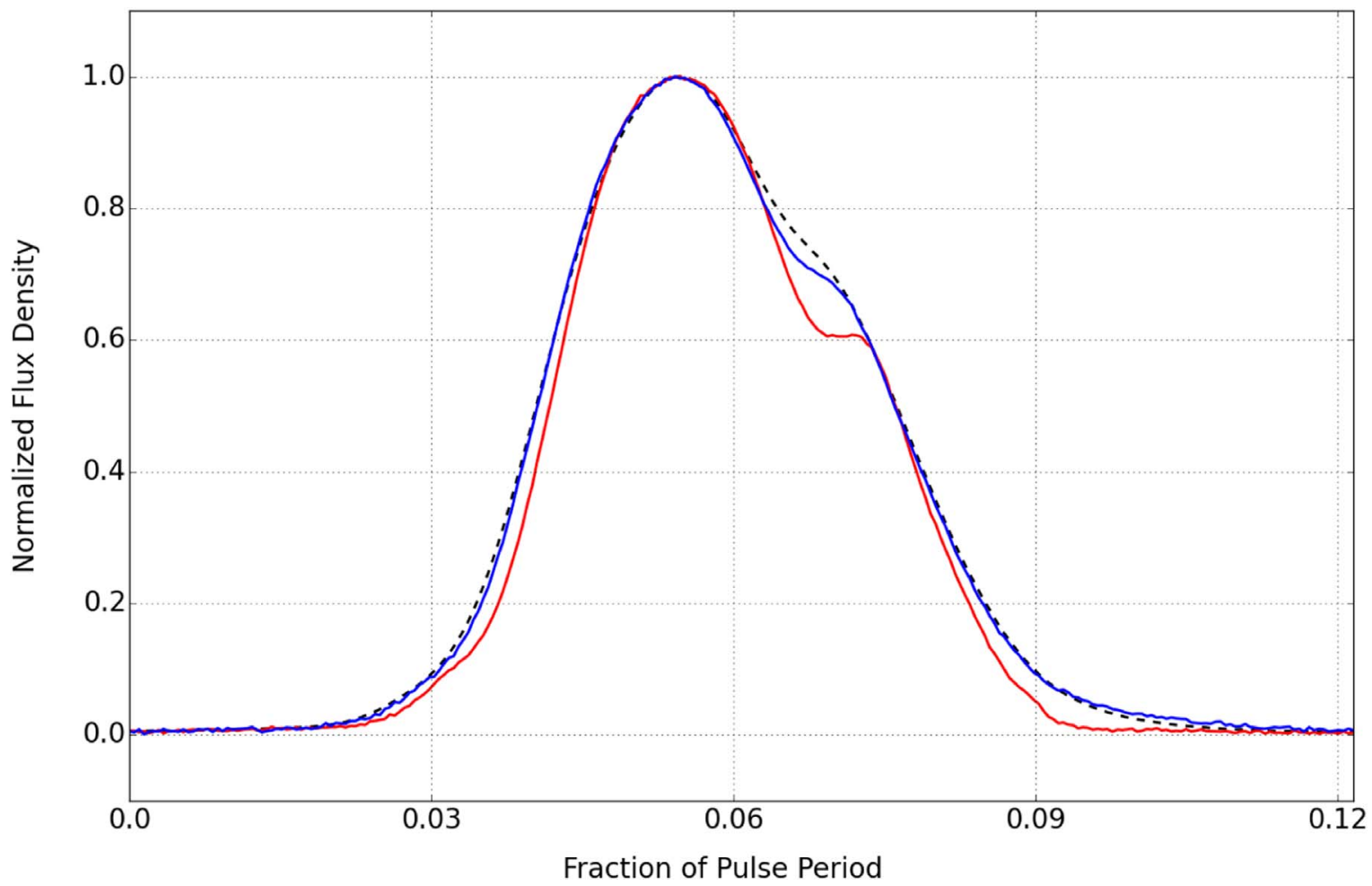

Figure 20. Scatter broadening simulation for PSR B1937+21. The red line is the $1400 \mathrm{MHz}$ AO pulse profile observed at MJD 55361, and the blue profile was observed on MJD 55828. The black dashed line is the result of a convolution of the red profile and a one-sided exponential function, in order to simulate the effects of scatter broadening. The $\tau$ value of the exponential function is $9.1 \mu \mathrm{s}$.

MJDs of 57083 (2015 March 2) and 57263 (2015 August 29), as shown in Figure 21. During this time there are various observations in which the shape of the frequency-integrated profiles and all of the contributing subbands is modified with respect to the average profile shape of the data set. Additionally, during this period there is a large fraction of observations in which the absolute fluxes are recorded as much larger than expected. The $\mathrm{S} / \mathrm{N}$ of these observations suggests that the high flux density is due to miscalibration rather than a very bright signal. Both of these phenomena are shown on MJD 57108 in Figure 22. The high concentration of pulse profile changes during this time period, along with their nature, may suggest an non-astrophysical cause. This hypothesis is bolstered by the fact that in the $2030 \mathrm{MHz}$ observations of PSR B1937+21, the most significant pulse profile variations also occur within this date range, as can be seen in Panel (B4) of Figure 5 and also in Figure 7. When analyzing all pulsar observations that appeared in the $2030 \mathrm{MHz}$ data set of PSR B1937+21 before any profiles were removed due to low $\mathrm{S} / \mathrm{N}$, three out of the four highest flux density observations fell within this MJD 57083-57263 range. The absolute fluxes of these observations are very large, with profile peaks around 27 , 44 , and $65 \mathrm{Jy}$, yet all have comparatively low $\mathrm{S} / \mathrm{N}$, as was the case for PSR J1713+0747. This span of time coincides with an era of particularly high levels of RFI around $2000 \mathrm{MHz}$ at AO. The RFI was eventually mitigated by a new filter installed by the observatory in October 2015. As part of the processing, extra RFI removal was carried out for all PUPPI $2030 \mathrm{MHz}$ data before MJD 57300. Much of the frequency band had to be removed from many of these observations, but residual effects of the RFI may well remain and be responsible for the AO profile changes at $2030 \mathrm{MHz}$.
Further information regarding the cause of these profile changes is provided when comparing the $2030 \mathrm{MHz}$ PSR B 1937+21 profiles occurring in the MJD 57083-57263 range (discussed previously; Figure 7) with Figure 23. As discussed in Section 2, only the $1500 \mathrm{MHz}$ GUPPI data has undergone two parallel methods of polarization calibration, using a noise diode and the more sophisticated full Mueller matrix calibration. This data set, therefore, gives us an opportunity to see how the different calibration techniques affect the resulting pulse profiles (see also Gentile et al. 2018). Only relatively subtle changes are produced by the different polarization calibration methods for most observations. However, there are some observation days that show large pulse profile variability when calibrated using only the noise diode. One such day is MJD 55977. Figure 23 shows the pulse profile modifications that take place in the PSR B1937+21 $1500 \mathrm{MHz}$ noise diode calibrated observations made on that day. These deviations from the average all but disappear when full Mueller matrix calibration is applied. The same phenomenon is seen on the same day for PSR J1713+0747.

The PUPPI $2030 \mathrm{MHz}$ profiles that fall in the problematic MJD 57083-57263 range and are highlighted in Figure 7 are very similar in nature to the GUPPI $1500 \mathrm{MHz}$ MJD 55977 profile that was polarization calibrated using only a noise diode, both in the main pulse and the interpulse. It is likely, therefore, that the $2030 \mathrm{MHz}$ PSR B1937+21 PUPPI profiles highlighted in Figure 7 are also the result of incorrect polarization calibration. Extrapolating further, the PSR J1713 $+07472030 \mathrm{MHz}$ PUPPI profile changes that also occur in the same problematic date range as the PSR B1937+21 $2030 \mathrm{MHz}$ PUPPI observations may also be due to incorrect polarization calibration. The polarimetric calibration of some NANOGrav MSPs is addressed in detail in Gentile et al. (2018). As 


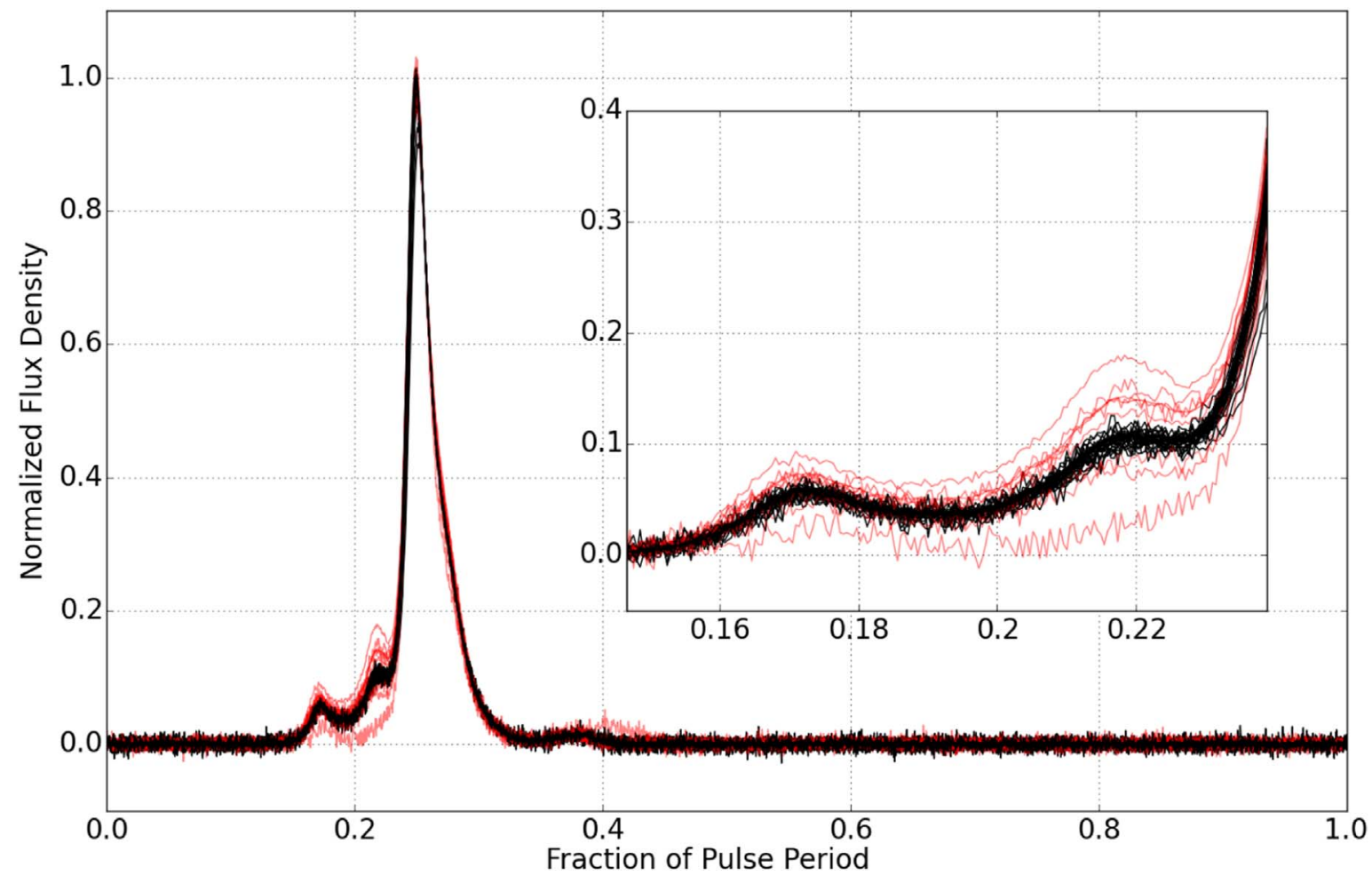

Figure 21. All 36 pulse profiles of PSR J1713+0747 observed at $2030 \mathrm{MHz}$ included in the variability analysis. The red profiles were observed on or between MJDs 57083 and 57263. All other profiles are black.

discussed in Section 2, Gentile et al. have performed full Mueller matrix polarization calibration for the PUPPI data. This is done using a method called Measurement Equation Template Matching (van Straten 2013), a technique that uses pulsars with known polarization profiles to act as standard sources in order to generate polarimetric responses for any epoch of observation. Unfortunately, the standard sources used by Gentile et al. were PSRs J1713+0747 and B1937+21. The polarization profiles for these two pulsars are, therefore, assumed to be unchanging and so are not calculated for each observation. In general, Gentile et al. find that the polarimetric responses of AO's 1400 and $2030 \mathrm{MHz}$ receivers vary significantly with time.

In general, it is possible that some pulse profile shape changes are the result of flux and polarization calibration issues. As discussed in Section 4.1, the flux density calibration procedure was not undertaken correctly for the $1400 \mathrm{MHz} \mathrm{AO}$ observation of PSR J1713+0747 made on MJD 56360; an incorrect pulsed calibration signal was injected at the epoch of observation. It is not clear whether the change in pulse profile shape was affected by this, as pulse profiles with similar shapes were also seen in the data set, for which no such calibration issues were seen (MJDs 56598 and 57239).

The profile shape changes of PSR J1643-1224 have now been observed by both the GBT and the Parkes Radio Telescope, and therefore, an instrumental cause can be ruled out.

\subsection{Jitter}

Pulsars are known to exhibit stochastic, broadband, singlepulse variations that are intrinsic to the pulsar emission process and affect the shape of the integrated pulse profile. This phenomenon is known as jitter and contributes noise to the TOAs. Cordes and Downs (1985) showed that on timescales ranging from one pulse period to integrations of up to an hour, TOA variations exceed what is expected from radiometer noise alone in long-period pulsars. Studies of MSPs show similar findings (e.g., Shannon et al. 2014), and this is generally true for NANOGrav MSPs (Lam et al. 2016b).

As jitter is expected to be uncorrelated from one pulse period to the next, it should not be responsible for any systematic profile changes, such as those seen in PSRs B1937+21 and J1643-1224 at $820 \mathrm{MHz}$. Using the AO $1400 \mathrm{MHz}$ receiver, Shannon and Cordes (2012) studied the impact that jitter has on the timing stability of PSR J1713+0747. They predict that for a 30 minute observation (comprising $\sim 10^{5.6}$ pulses), jitter will produce a scatter $\sigma_{\mathrm{J}}$ in the arrival times of $\sim 40 \mathrm{~ns}$. Similarly, Lam et al. (2016b) calculate $\sigma_{\mathrm{J}}$ for pulsars in the NANOGrav 9year data set and find values for PSR J1713+0747 that range from $39 \mathrm{~ns}$ in the AO $1400 \mathrm{MHz}$ data to $91 \mathrm{~ns}$ in the GBT $820 \mathrm{MHz}$ data. They find $\sigma_{\mathrm{J}}$ for PSR B1937+21 to be between $5.7 \mathrm{~ns}(\mathrm{AO} 1400 \mathrm{MHz}$ ) and $32 \mathrm{~ns}(\mathrm{GBT} 820 \mathrm{MHz})$. For PSR J2145-0750, $\sigma_{\mathrm{J}}$ is calculated to be $89 \mathrm{~ns}$ at $820 \mathrm{MHz}$ and $120 \mathrm{~ns}$ at $1500 \mathrm{MHz}$ using GBT data. These values are much smaller than the changes in TOA induced by the observed changes in pulse profile, as seen in Table 2. This indicates that pulse jitter is not the dominant source of the profile changes we observe in PSRs J1713+0747, B1937+21, and J2145-0750. Furthermore, many other pulsars observed by the NANOGrav collaboration show evidence of more jitter noise but less profile shape variability.

Lam et al. (2016b) calculate $\sigma_{\mathrm{J}}$ for PSR J1643-1224 as 162 and $219 \mathrm{~ns}$ at 820 and $1500 \mathrm{MHz}$, respectively. This is the same order of magnitude as the changes in TOA induced by the observed changes in pulse profile (Table 2). However, the 

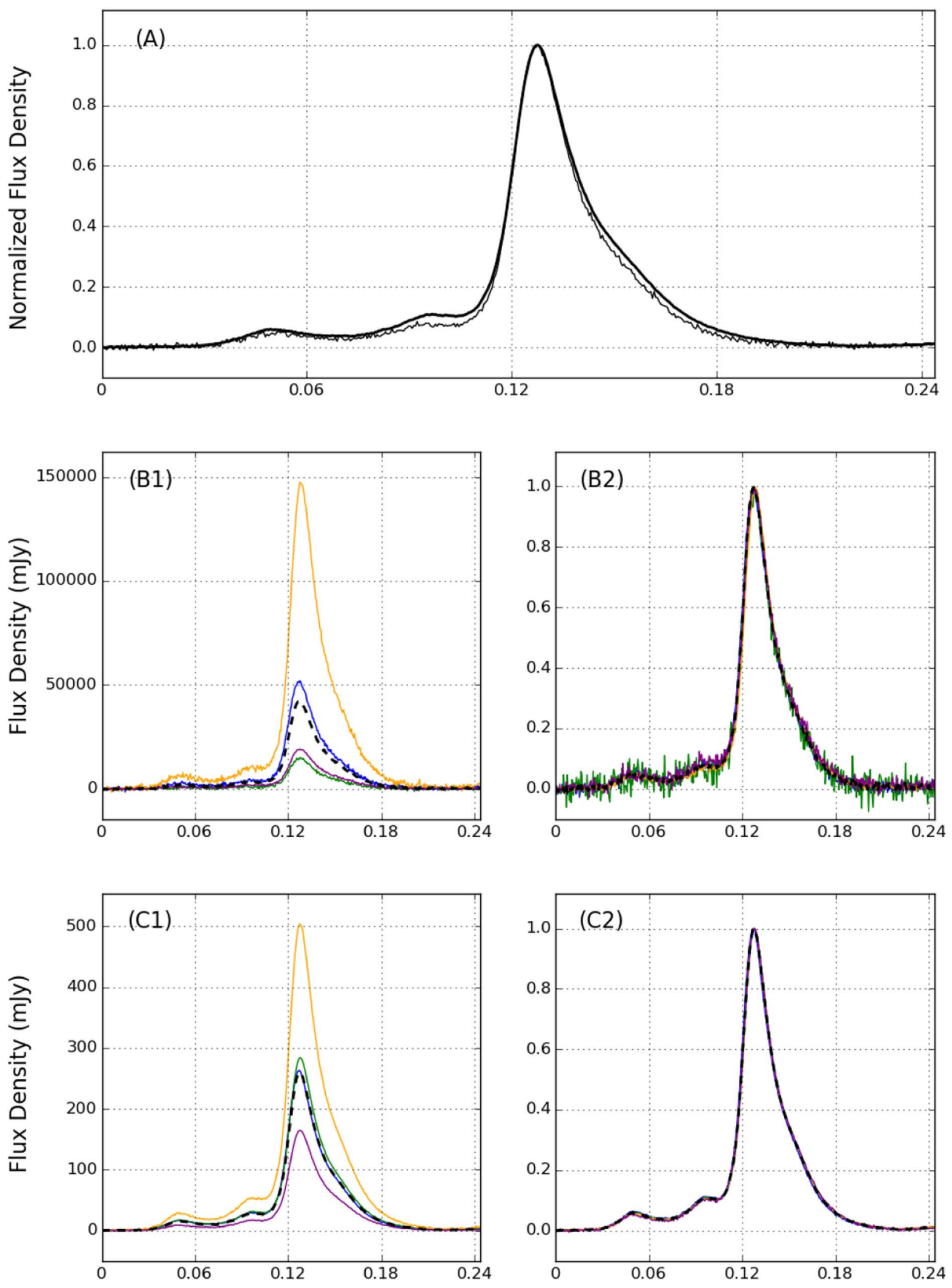

Fraction of Pulse Period

Figure 22. Two contrasting pulse profiles of PSR J1713+0747 at $2030 \mathrm{MHz}$. The thin line in Panel (A) and Panels (B1) and (B2) show the observation made on MJD 57108. The thick line and Panels (C1) and (C2) show the observation made on MJD 57375. The plots are as Figure 17 otherwise. A comparison of Panels (B2) and (C2) shows that the shapes of the frequency-integrated profiles and all of the contributing subbands are different for the two observations. This is most noticeable at a pulse phase fraction $\sim 0.10$. The absolute flux in Panel (B1) is recorded as much larger than expected. 

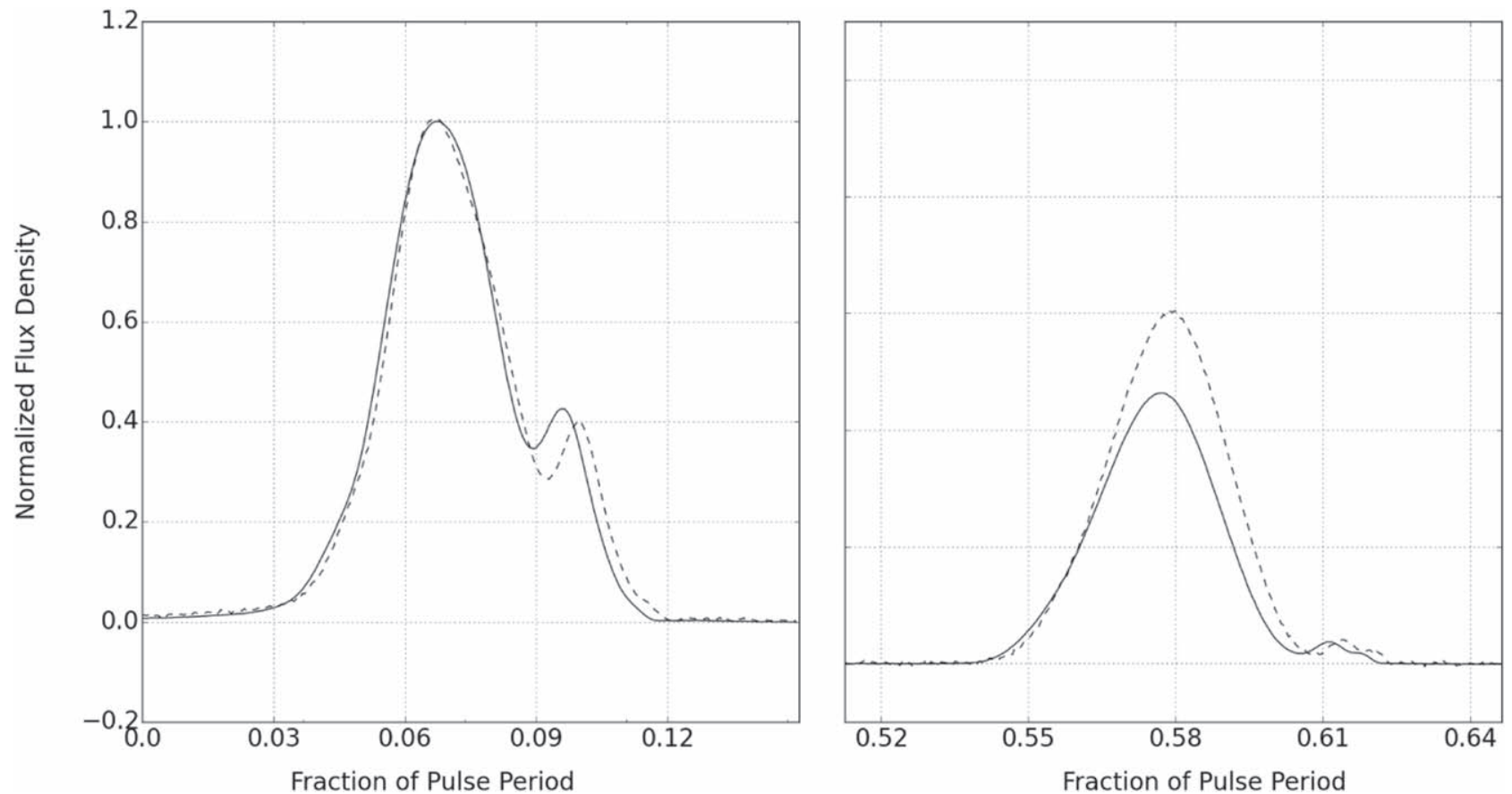

Figure 23. Pulse profile deviations from PSR B1937+21 $1500 \mathrm{MHz}$ GBT observations that seem to result from incorrect polarization calibration. The solid line shows the average profile for the data set. The dashed profile is an MJD 55977 observation that has had noise diode polarization calibration applied. This discrepancy is not present when full Mueller matrix calibration is employed.

drifting and systematic nature of the profile changes in the data set is not indicative of jitter, which is uncorrelated in time.

\subsection{Other Pulsar Emission Changes}

Some pulse profile variability observed in PSRs J2145 -0750 and B1937+21 is consistent with effects of the propagation of a radio signal through the IISM-scintillation and scatter broadening, respectively. Some profile modulations in PSRs J1713+0747 and B1937+21 may also be the product of improper polarization calibration. Other pulse profile shape changes elude a comprehensive explanation and so emission changes intrinsic to the pulsar (besides jitter) cannot be ruled out.

As described above, the changes in PSR J1643-1224 profile do not seem to be characteristic of modulations induced by propagation effects, inaccurate DMs, jitter, or instrumental issues. As pointed out by Shannon et al. (2016), the drifting nature of pulse profile disturbances is reminiscent of that seen in PSR J0738 -4042 - a pulsar displaying simultaneous changes in emission and rotation, which were assessed to be intrinsic to the neutron star (Brook et al. 2014).

We also note here that PSR B1937+21 is known to emit giant pulses (Cognard et al. 1996). The longitude at which the giant pulses are seen to occur is not consistent with the pulse profile shape changes that we see. Additionally, the profile variability in PSR B1937+21 occurs on timescales of hundreds of days; no such timescale is known for giant pulse activity.

In general, there are few obvious correlations between the profile shape changes and pulsar flux density (as seen by comparing the (A) and (B) prefixed panels in the variability maps). The notable exception is the period between MJDs 57083 and 57263 at $2030 \mathrm{MHz}$ in PSRs J1713+0747, B1937+21, as discussed in Section 5.6.

Other links between the profile variability and the rotational behavior of a pulsar may provide further clues regarding the source of any variability. Figure 24 shows the behavior of both profile and timing residuals for PSR B1937+21. The profile residuals shown are at an observing frequency of $820 \mathrm{MHz}$ (the data set displaying the most systematic variability). A more detailed analysis of any relationship between the emission and rotational properties of these pulsars will be left to future work.

Whatever the cause of unmodeled pulse profile changes, they are detrimental to the template matching technique of TOA determination and, therefore, to pulsar timing. For PSRs J1643 $-1224, \mathrm{~J} 1713+0747, \mathrm{~B} 1937+21$, and $\mathrm{J} 2145-0750$, the TOA inaccuracies induced due to some changes in pulse profile are on the order of hundreds of nanoseconds to microseconds. The frequency-integrated pulse profile changes that we have focused on may not translate to profile changes in the narrow individual frequency channels that the NANOGrav collaboration uses to produce its TOAs, however; pulse profiles that result from the combination of a relatively wide band of frequency channels are far more sensitive to shape changes induced by the effects of signal propagation. It is also important that highly aberrant pulse profiles that appear in a data set have their corresponding TOAs removed in order to ensure that the most accurate timing models are produced.

When looking at figures that show the phase location of profile variability, we must be cognizant of the fact that different methods for alignment will show the variability to occur at different parts of the pulse profile. Different alignment methods, however, should largely be in agreement regarding the amount of variability that is contained within a data set, even if they disagree on the phases at which it occurs. A priori, we expect the magnitude of profile variability to be most around the profile peak if we assume that the amount of variability will be proportional to the profile intensity at the phase at which it occurs.

When quantifying the variability seen in pulse profiles, we have discounted the number of pulsar rotations that contribute 


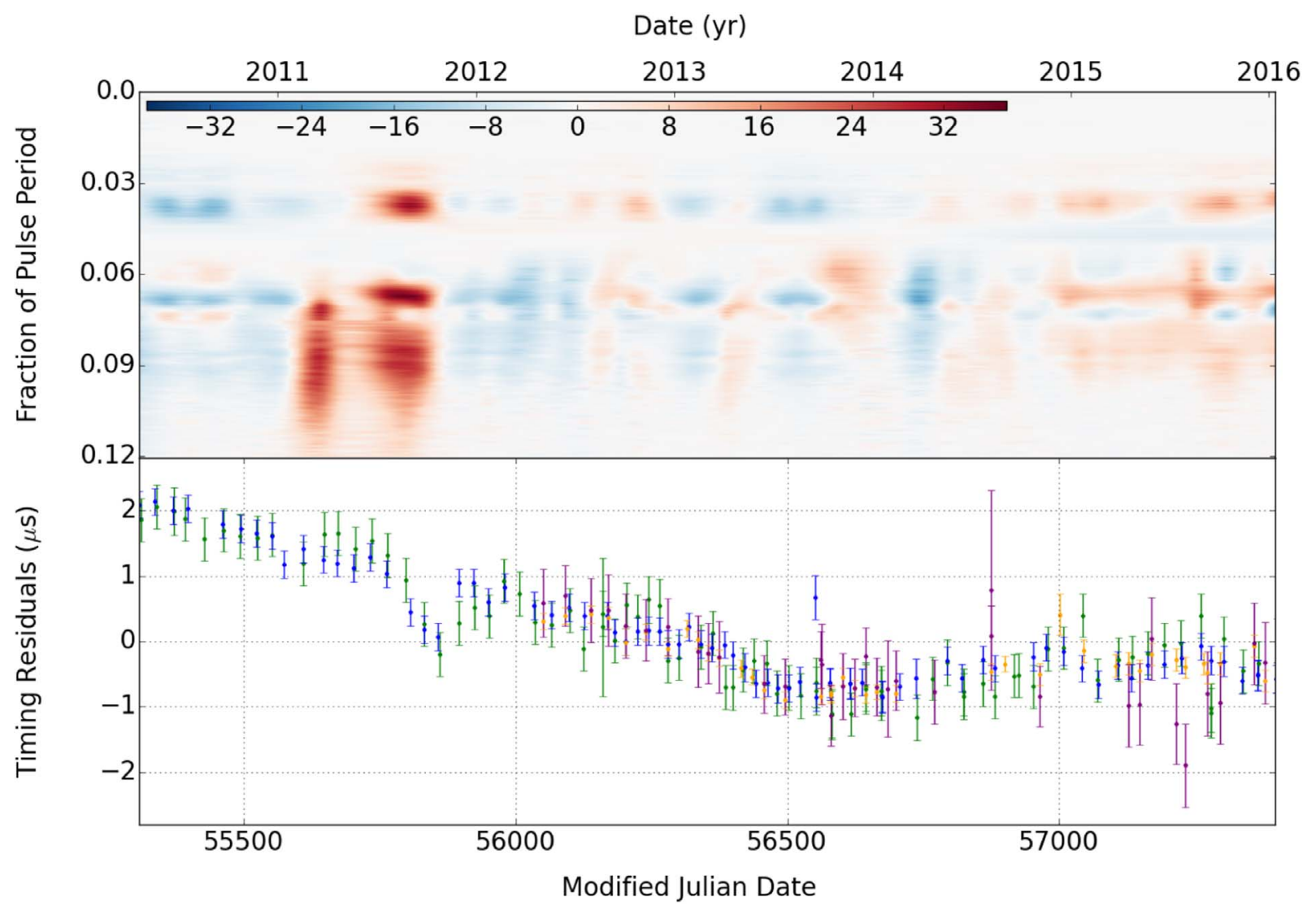

Figure 24. $820 \mathrm{MHz}$ profile residuals and timing residuals of B1937+21, observed at the GBT. The top panel is a variability map showing pulse profile shape changes after the observations have been normalized and depicts the same data as Panel (B1) of Figure 5. Red regions indicate where the inferred pulse profile has an excess of flux density compared to the average for the data set. Blue indicates where it has a deficit. The unit for the variability map is the mean of the standard deviation of the off-pulse phase bins for the data set. The bottom panel shows the TOA residuals for PSR B1937-21 at $820 \mathrm{MHz}$ (green), $1400 \mathrm{MHz}$ (orange), $1500 \mathrm{MHz}$ (blue), and $2030 \mathrm{MHz}$ (purple).

to the observations. With its very short period of $1.56 \mathrm{~ms}$, the PSR B1937+21 data sets typically have around $10^{6}$ rotations per observation. Conversely, PSR J2145-0750 has the longest period of all pulsars analyzed in this work at $16.05 \mathrm{~ms}$. Consequently, the data sets for this pulsar have only around $10^{5}$ rotations per observation. All else being equal, 10 times more pulses contributing to an integrated pulse profile would increase the $\mathrm{S} / \mathrm{N}$ by approximately $\sqrt{10}$ and would also decrease the pulse profile variability due to jitter, thereby decreasing the variability measured. Falling in between these extremes, PSRs J1643-1224 and J1713+0747 have pulse periods of 4.62 and $4.57 \mathrm{~ms}$, respectively, and so rotate approximately $3-4 \times 10^{5}$ times per observation.

In future work, MSP pulse profile variability information could lead to the mitigation of timing aberrations caused by the unmodeled pulse profile changes we observe. For example, more NANOGrav data are currently undergoing full Mueller matrix polarization calibration; we have shown that this process can correct pulse profile shape distortions that may result from imperfect calibration when using only a local noise diode. In the case of a pulsar in which pulse profile variability is primarily due to temporal broadening from scattering, we can apply techniques such as cyclic spectroscopy to recover the intrinsic pulse profiles (Demorest 2011; Walker et al. 2013) from the effects of interstellar scattering. In these two examples, as the differences between the shape of the observed profiles and the timing template are reduced, so too are the timing residuals. If profile shape changes are entirely due to
DISS, then the consequences for timing can be minimized by calculating the TOAs for relatively narrow frequency subbands, as is already done by NANOGrav.

To create the smooth, continuous variability maps seen throughout this paper, we have inferred the behavior of the flux density for each phase bin (and, therefore, of the pulse profile as a whole) between observations using GP regression. For pulsars that show systematic variability, such modeling techniques would also permit the extrapolation of pulse profiles shapes. A predicted profile shape could then be used as a dynamic template for the TOA calculation. Using an accurate template shape (if one can be calculated) will necessarily also improve the accuracy of the TOA recorded. For pulsars with more erratic shape changes and less systematic variability, such extrapolations will be difficult to make. However, throughout this analysis, we have also used a new pulse profile alignment technique which maximizes the number of pulse phase bins that are in agreement (see Section 3.1 for details). As a result, only the stable parts of the pulse profiles are used in their alignment. Using only these stable phase bins in the template matching procedure could potentially result in reduced timing residuals for some pulsar data sets.

The question of how the variability measured in this work will impact the predicted timeline for nanohertz gravitational wave detection is a difficult one. Relatively little research has been done on long-term pulse profile variability in MSPs. The physical origin of much of the emerging profile variability is uncertain, can be different for each data set analyzed, and must 


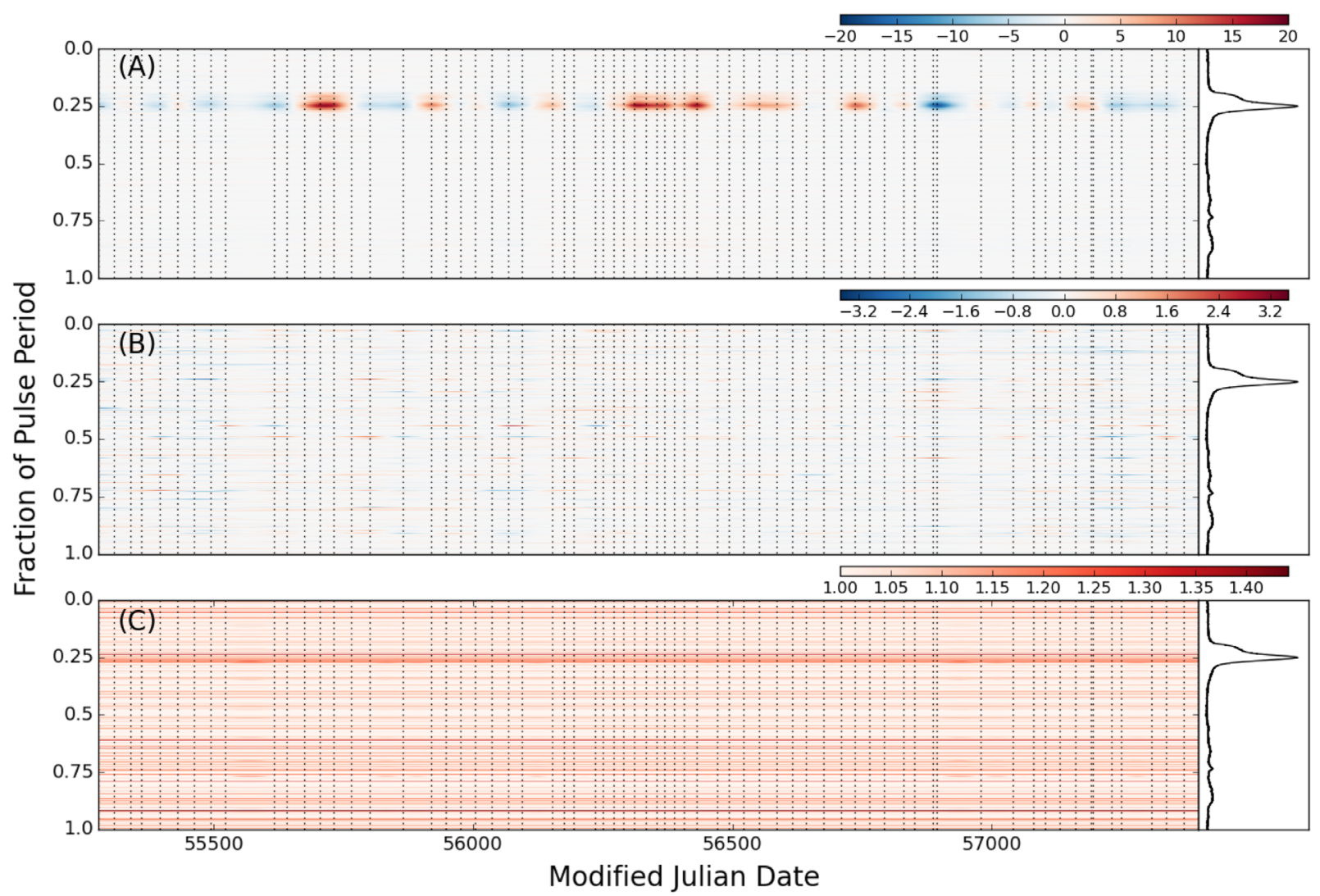

Figure 25. Variability map for a data set in which the pulse profile shape is relatively stable. Panel (A) shows the flux density variations in the flux-calibrated, prenormalized observations of PSR J1614-2230 at $820 \mathrm{MHz}$. The flux density variations are due to refractive and diffractive scintillation. Red regions indicate where the inferred pulse profile has an excess of flux density compared to the average for the data set. Blue indicates where it has a deficit. Most variability is seen around the peak of the profile at a pulse period fraction of $\sim 0.25$. Panel (B) shows any pulse profile shape changes after the observations have been normalized. As any variability in this data set is consistent with additive white Gaussian noise, the variability map is almost entirely devoid of color; in each phase bin, the GP model lies around zero (i.e., the mean of the data points which inform it). Panel (C) shows the standard deviation of the inferred GP model as a function of pulse phase and time. The unit for all panels is the mean of the standard deviation of the off-pulse phase bins for the data set. In Panel (C) the value is close to unity across the whole pulse profile; the variance of the data is approximately the same whether looking at on- or off-pulse phase bins. The vertical dotted lines indicate the epochs of observation informing the GP models. To the right of each panel, the average pulse profile for the data set is shown.

be a mixture of multiple effects to varying degrees. Mitigation of profile variability will require further investigation, and therefore, it is not clear how soon we will be able to accommodate such profile changes in a pulsar timing model. As evidence for MSP profile variability grows, so too will the voicing of suggestions that precision pulsar timing should not be done using the standard template matching techniques but instead, using other techniques that are more accommodating to such variability (e.g., the profile domain pulsar timing analysis of Lentati et al. 2015). Such discussions make the analyses in this paper more interesting and relevant.

\section{Conclusions}

The primary aim of this work was to analyze the long-term pulse profile behavior in the 11-year data set employed by the NANOGrav collaboration to search for nanohertz frequency gravitational waves; significant profile variability is detrimental to the effort if overlooked.

PSRs J1713+0747, B1937+21, and J2145-0750 show the highest levels of variability of the pulsars analyzed, with PSR B1937+21 showing significant long-timescale trends. These pulsars are also three of the brightest observed by the NANOGrav collaboration. This is not entirely surprising, as any pulse profile shape changes are more easily classified as such in bright pulsars, and also the variability metric is in units of the rms levels of the off-pulse regions, which will be relatively small in such pulsars. Despite this, some of the profile changes seen in these pulsars are of a magnitude that means they would also be visible in pulsars with a much lower $\mathrm{S} / \mathrm{N}$; the method used for detecting long-term variability has been shown to be able to do so down to a level that is comparable in magnitude to the rms of the observation noise (Brook et al. 2016). Systematic variability is also observed in the PSR J1643-1224 data, which has been identified previously in observations by the Parkes radio telescope. The cause is not yet clear beyond being astrophysical in nature. The variability seen in PSR J2145-0750 is consistent with scintillation effects. Some of the profile modification seen in PSRs J1713+0747 and B1937+21 is likely due to improper polarization calibration, and in the $2030 \mathrm{MHz}$ AO observations, RFI is suspected to have strongly influenced some of the changes. However, some variability in B1937+21 also seems consistent with scatter broadening, while some profile changes in PSRs J1713+0747 are due to effects of scintillation.

In the future, the impact of pulse profile variability on precision timing can be minimized by techniques such as full Mueller matrix polarization calibration, cyclic spectroscopy, the employment of dynamic templates in the template matching procedure, and timing to the most stable parts of a pulse profile. 
The NANOGrav project is supported by NSF Physics Frontiers Center award PHYS-1430284. P.R.B. is supported by Track I award OIA-1458952. The Green Bank Observatory is a facility of the National Science Foundation operated under cooperative agreement by Associated Universities, Inc. The Arecibo Observatory is operated by SRI International under a cooperative agreement with the NSF (AST-11000968), and in alliance with the Ana G. Méndez-Universidad Metropolitana and the Universities Space Research Association. Pulsar research at the University of British Columbia is supported by an NSERC Discovery Grant and by the Canadian Institute for Advanced Research. J.A.E. was partially supported by NASA through Einstein Fellowship grant PF4-150120. W.W.Z. is supported by the CAS Pioneer Hundred Talents Program and the Strategic Priority Research Program of the Chinese Academy of Sciences grant No. XDB23000000. Portions of this work performed at NRL were supported by the Chief of Naval Research.

\section{Appendix \\ A Variability Map Demonstrating a Stable Pulse Profile Shape}

For illustrative purposes, we present in Figure 25 a variability map for a pulsar that has a stable pulse profile.

\section{ORCID iDs}

P. R. Brook (iD https://orcid.org/0000-0003-3053-6538

M. A. McLaughlin (D) https://orcid.org/0000-0001-7697-7422

M. T. Lam (1) https://orcid.org/0000-0003-0721-651X

S. Chatterjee (iD https://orcid.org/0000-0002-2878-1502

J. M. Cordes (iD https://orcid.org/0000-0002-4049-1882

M. DeCesar (10 https://orcid.org/0000-0002-2185-1790

T. Dolch (i) https://orcid.org/0000-0001-8885-6388

R. D. Ferdman (ib https://orcid.org/0000-0002-2223-1235

P. A. Gentile (iD https://orcid.org/0000-0001-8158-683X

M. L. Jones (1D https://orcid.org/0000-0001-6607-3710

L. Levin (i) https://orcid.org/0000-0002-2034-2986

D. R. Lorimer (iD https://orcid.org/0000-0003-1301-966X

R. S. Lynch (1) https://orcid.org/0000-0001-5229-7430

D. J. Nice (i) https://orcid.org/0000-0002-6709-2566

T. T. Pennucci (iD https://orcid.org/0000-0001-5465-2889

S. M. Ransom (ib https://orcid.org/0000-0001-5799-9714

P. S. Ray (i) https://orcid.org/0000-0002-5297-5278

R. Spiewak (i) https://orcid.org/0000-0002-6730-3298

I. H. Stairs (iD https://orcid.org/0000-0001-9784-8670

D. R. Stinebring (i) https://orcid.org/0000-0002-1797-3277

K. Stovall (iD https://orcid.org/0000-0002-7261-594X

J. K. Swiggum (iD https://orcid.org/0000-0002-1075-3837

W. W. Zhu (i) https://orcid.org/0000-0001-5105-4058

\section{References}

Armstrong, J. W. 1984, Natur, 307, 527

Arzoumanian, Z., Brazier, A., Burke-Spolaor, S., et al. 2015, ApJ, 813, 65 Arzoumanian, Z., Brazier, A., Burke-Spolaor, S., et al. 2018, ApJS, 235, 37
Backer, D. C. 1970a, Natur, 228, 42

Backer, D. C. 1970b, Natur, 228, 1297

Backer, D. C., Kulkarni, S. R., Heiles, C., Davis, M. M., \& Goss, W. M. 1982, Natur, 300, 615

Backer, D. C., Wong, T., \& Valanju, J. 2000, ApJ, 543, 740

Brook, P. R., Karastergiou, A., Buchner, S., et al. 2014, ApJL, 780, L31

Brook, P. R., Karastergiou, A., Johnston, S., et al. 2016, MNRAS, 456, 1374

Camilo, F., Ransom, S. M., Chatterjee, S., Johnston, S., \& Demorest, P. 2012, ApJ, 746, 63

Cognard, I., Bourgois, G., Lestrade, J.-F., et al. 1995, A\&A, 296, 169

Cognard, I., Shrauner, J. A., Taylor, J. H., \& Thorsett, S. E. 1996, ApJL, 457, L81

Cordes, J. M., \& Downs, G. S. 1985, ApJS, 59, 343

Cordes, J. M., \& Lazio, T. J. 1991, ApJ, 376, 123

Cordes, J. M., \& Rickett, B. J. 1998, ApJ, 507, 846

Cordes, J. M., Wolszczan, A., Dewey, R. J., Blaskiewicz, M., \& Stinebring, D. R. 1990, ApJ, 349, 245

Demorest, P. B. 2011, MNRAS, 416, 2821

DuPlain, R., Ransom, S., Demorest, P., et al. 2008, Proc. SPIE, 7019, 70191D Ford, J. M., Demorest, P., \& Ransom, S. 2010, Proc. SPIE, 7740, 77400A

Gentile, P. A., McLaughlin, M. A., Demorest, P. B., et al. 2018, ApJ, 862, 47 Graham Smith, F., Lyne, A. G., \& Jordan, C. 2011, MNRAS, 410, 499

Heiles, C., Perillat, P., Nolan, M., et al. 2001, PASP, 113, 1274

Helfand, D. J., Manchester, R. N., \& Taylor, J. H. 1975, ApJ, 198, 661 Hobbs, G. 2013, CQGra, 30, 224007

Hotan, A. W., Bailes, M., \& Ord, S. M. 2005, ApJ, 624, 906

Jones, M. L., McLaughlin, M. A., Lam, M. T., et al. 2017, ApJ, 841, 125

Karastergiou, A., Roberts, S. J., Johnston, S., et al. 2011, MNRAS, 415, 251

Kaspi, V. M., Taylor, J. H., \& Ryba, M. F. 1994, ApJ, 428, 713

Keith, M. J., Coles, W., Shannon, R. M., et al. 2013, MNRAS, 429, 2161

Kramer, M. 1998, ApJ, 509, 856

Kramer, M., \& Champion, D. J. 2013, CQGra, 30, 224009

Kramer, M., Lyne, A. G., O’Brien, J. T., Jordan, C. A., \& Lorimer, D. R. 2006 , Sci, 312, 549

Lam, M. T. 2017, PyPulse: PSRFITS handler, Astrophysics Source Code Library, ascl:1706.011

Lam, M. T., Cordes, J. M., Chatterjee, S., et al. 2016a, ApJ, 821, 66

Lam, M. T., Cordes, J. M., Chatterjee, S., et al. 2016b, ApJ, 819, 155

Lentati, L., Alexander, P., \& Hobson, M. P. 2015, MNRAS, 447, 2159

Levin, L., McLaughlin, M. A., Jones, G., et al. 2016, ApJ, 818, 166

Lorimer, D. R., Lyne, A. G., McLaughlin, M. A., et al. 2012, ApJ, 758, 141

Lyne, A., Hobbs, G., Kramer, M., Stairs, I., \& Stappers, B. 2010, Sci, 329, 408

Lyne, A. G., Smith, F. G., \& Graham, D. A. 1971, MNRAS, 153, 337

Lyne, A. G., Stappers, B. W., Freire, P. C. C., et al. 2017, ApJ, 834, 72

Manchester, R. N., Hobbs, G., Bailes, M., et al. 2013, PASA, 30, e017

McLaughlin, M. A. 2013, CQGra, 30, 224008

Michilli, D., Hessels, J. W. T., Donner, J. Y., et al. 2018, MNRAS, 476, 2704

Pennucci, T. T., Demorest, P. B., \& Ransom, S. M. 2014, ApJ, 790, 93

Ramachandran, R., Demorest, P., Backer, D. C., Cognard, I., \& Lommen, A. 2006, ApJ, 645, 303

Rasmussen, C. 2006, Gaussian Processes for Machine Learning (Cambridge, MA: MIT Press)

Rathnasree, N., \& Rankin, J. M. 1995, ApJ, 452, 814

Rickett, B. J. 1990, ARA\&A, 28, 561

Roberts, S., Osborne, M., Ebden, M., et al. 2012, RSPTA, 371, 1984

Shannon, R. M., \& Cordes, J. M. 2010, ApJ, 725, 1607

Shannon, R. M., \& Cordes, J. M. 2012, ApJ, 761, 64

Shannon, R. M., Cordes, J. M., Metcalfe, T. S., et al. 2013, ApJ, 766, 5

Shannon, R. M., Lentati, L. T., Kerr, M., et al. 2016, ApJL, 828, L1

Shannon, R. M., Osłowski, S., Dai, S., et al. 2014, MNRAS, 443, 1463

Stairs, I. H., Lyne, A. G., \& Shemar, S. L. 2000, Natur, 406, 484

Taylor, J. H. 1992, RSPTA, 341, 117

Taylor, J. H., Manchester, R. N., \& Huguenin, G. R. 1975, ApJ, 195, 513

van Straten, W. 2004, ApJS, 152, 129

van Straten, W. 2006, ApJ, 642, 1004

van Straten, W. 2013, ApJS, 204, 13

Walker, M. A., Demorest, P. B., \& van Straten, W. 2013, ApJ, 779, 99 